\title{
Physical characterization of S169: a prototypical IR bubble associated with the massive star-forming region IRAS 12326-6245
}

\author{
N. U. Duronea ${ }^{1, \star}$, S. Cichowolski ${ }^{2, \star}$, L. Bronfman ${ }^{3}$, E. Mendoza ${ }^{4}$, R. Finger ${ }^{3}$, L. A. Suad ${ }^{2, \star}$, \\ M. Corti ${ }^{5,6, \star}$, and E. M. Reynoso ${ }^{2, \star}$
}

\author{
${ }^{1}$ Instituto de Astrofísica de La Plata (UNLP - CONICET), La Plata, Argentina \\ e-mail: nduronea@fcaglp.unlp.edu.ar \\ 2 Instituto de Astronomía y Física del Espacio (UBA, CONICET), CC 67, Suc. 28, 1428 Buenos Aires, Argentina \\ ${ }^{3}$ Departamento de Astronomía, Universidad de Chile, Casilla 36, Santiago de Chile, Chile \\ ${ }^{4}$ Observatório do Valongo, Universidade Federal do Rio de Janeiro, Ladeira Pedro Antônio, 43, Rio de Janeiro 20080-090, Brazil \\ ${ }^{5}$ Facultad de Ciencias Astronómicas y Geofísicas, Universidad Nacional de La Plata, Paseo del Bosque s/n, 1900 La Plata, \\ Argentina \\ ${ }^{6}$ Instituto Argentino de Radioastronomía (CCT-La Plata, CONICET; CICPBA), C.C. No. 5, 1894 Villa Elisa, Argentina
}

Received 31 July 2020 / Accepted 3 December 2020

\begin{abstract}
Aims. With the aim of studying the physical properties of Galactic IR bubbles and to explore their impact in massive star formation, we present a study of the IR bubble S169, associated with the massive star forming region IRAS 12326-6245.

Methods. We used $\mathrm{CO}(2-1),{ }^{13} \mathrm{CO}(2-1), \mathrm{C}^{18} \mathrm{O}(2-1), \mathrm{HCN}(3-2)$, and $\mathrm{HCO}^{+}(3-2)$ line data obtained with the APEX telescope using the on-the-fly full sampling technique to study the properties of the molecular gas in the nebula and the IRAS source. To analyze the properties and distribution of the dust, we made use of images obtained from the IRAC-GLIMPSE, Herschel, and ATLASGAL archives. The properties of the ionized gas in the nebula were studied using radio continuum and $\mathrm{H} \alpha$ images obtained from the SUMSS survey and SuperCOSMOS database, respectively. In our search for stellar and protostellar objects in the region, we used point source calalogs obtained from the MSX, WISE, GLIMPSE, 2MASS, AAVSO, ASCC-2.5V3, and GAIA databases.

Results. The new APEX observations allowed us to identify three molecular components, each one associated with different regions of the nebula, namely: at $-39 \mathrm{~km} \mathrm{~s}^{-1}$ (component A), $-25 \mathrm{~km} \mathrm{~s}^{-1}$ (component B), and $-17 \mathrm{~km} \mathrm{~s}^{-1}$ (component C). Component A is shown to be the most dense and clumpy. Six molecular condensations (MC1 to MC6) were identified in this component, with MC3 (the densest and more massive one) being the molecular counterpart of IRAS 12326-6245. For this source, we estimated an $\mathrm{H}_{2}$ column density up to $8 \times 10^{23} \mathrm{~cm}^{-2}$. An LTE analysis of the high density tracer lines $\mathrm{HCO}^{+}(3-2)$ and $\mathrm{HCN}(3-2)$ on this source, assuming 50 and $150 \mathrm{~K}$, respectively, indicates column densities of $N\left(\mathrm{HCO}^{+}\right)=(5.2 \pm 0.1) \times 10^{13} \mathrm{~cm}^{-2}$ and $N(\mathrm{HCN})=(1.9 \pm 0.5) \times$ $10^{14} \mathrm{~cm}^{-2}$. To explain the morphology and velocity of components $\mathrm{A}, \mathrm{B}$, and $\mathrm{C}$, we propose a simple model consisting of a partially complete semisphere-like structure expanding at $\sim 12 \mathrm{~km} \mathrm{~s}^{-1}$. The introduction of this model has led to a discussion about the distance to both S169 and IRAS 12326-6245, which was estimated to be $\sim 2 \mathrm{kpc}$. Several candidate YSOs were identified, projected mostly onto the molecular condensations MC3, MC4, and MC5, which indicates that the star-formation process is very active at the borders of the nebula. A comparison between observable and modeled parameters was not enough to discern whether the collect-and-collapse mechanism is acting at the edge of S169. However, other processes such as radiative-driven implosion or even a combination of both mechanisms, namely, collect-and-collapse and radiative-driven implosion, could be acting simultaneously in the region.
\end{abstract}

Key words. ISM: bubbles - HII regions - ISM: molecules - ISM: kinematics and dynamics

\section{Introduction}

Massive stars modify the interstellar medium (ISM), where they are born through their stellar winds and UV photons, creating interstellar bubbles and HII regions. These nebulae are detected in the optical and radio continuum ranges, showing the presence of ionized gas, and at infrared (IR), millimeter, and submillimeter wavelengths, indicating the presence of both dust (at different temperatures) and molecules.

Milky Way surveys at IR wavelengths have provided a plethora of examples of bubbles/HII regions, where their physical properties can be obtained on pc/sub-pc scales. Making use of the $8.0 \mu \mathrm{m}$ Spitzer-GLIMPSE survey, Benjamin et al. (2003)

\footnotetext{
^ Member of the Carrera del Investigador Científico of CONICET, Argentina.
}

and Churchwell et al. $(2006,2007)$ identified more than 600 candidates for interstellar IR dust bubbles between Galactic longitudes of $-60^{\circ}$ to $60^{\circ}$. Follow-up observations of the stellar and prestellar populations around some of these bubbles have begun to link them with sites of recent triggered massive star formation (e.g., Zavagno et al. 2006; Watson et al. 2008; Deharveng et al. 2008, 2009; Samal et al. 2014; Kendrew et al. 2016; Duronea et al. 2017). These kinds of studies provide important insights into the evolution of interstellar bubbles, the characteristics of the interstellar medium where they evolve, the role of massive stars on favoring or suppressing new generations of stars, and the physical conditions under which massive stars may be induced to form. As part of a project aimed at characterizing and studying the physical properties of Galactic IR bubbles and their surroundings, and to better understand their influence in 


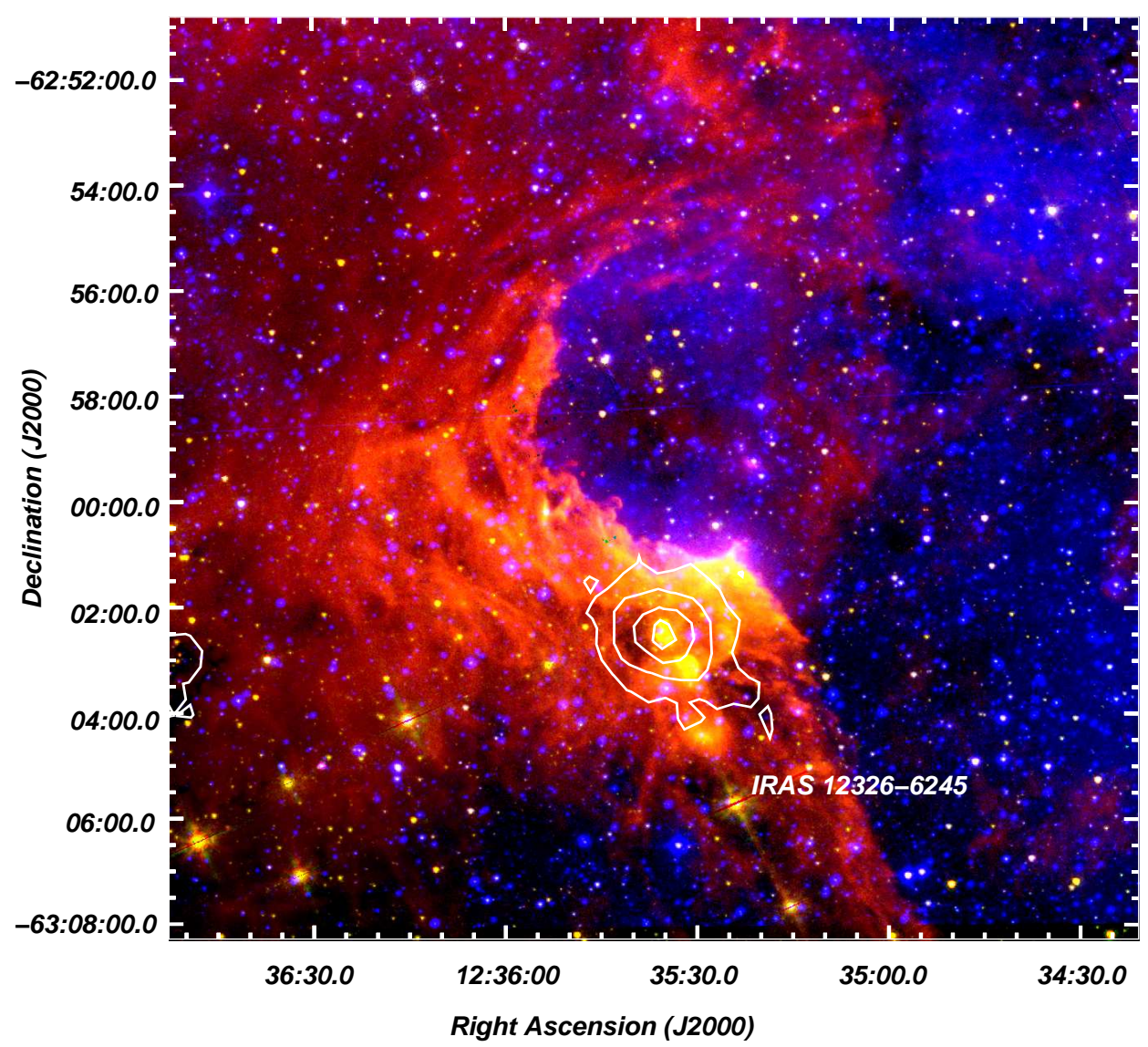

Fig. 1. Composite image of S169 and its environs showing the (IRAC-GLIMPSE) emissions at 8.0 (red) and $5.8 \mu \mathrm{m}$ (green). The superCOSMOS $\mathrm{H} \alpha$ emission is in blue. White contours depict the continuum emission at $870 \mu \mathrm{m}$ (ATLASGAL) arising from IRAS 12326-6245. massive star formation, we selected a southern field containing S169 (Churchwell et al. 2006), which is a prototypical IR bubble-shaped nebula of about 8 arcmin in diameter, centered at RA, Dec $(J 2000)=\left(12^{\mathrm{h}} 35^{\mathrm{m}} 29^{\mathrm{s}} .2,-62^{\circ} 58^{\prime} 03^{\prime \prime} .8\right)$. Figure 1 shows a composite image of S169 in 8.0 and $5.8 \mu \mathrm{m}$ (IRAC-GLIMPSE) and the $\mathrm{H} \alpha$ (superCOSMOS) emissions. The presence of emission in the $8-\mu \mathrm{m}$ band, mostly dominated by the emission of polycyclic aromatic hydrocarbons molecules (PAHs) at 7.7 and $8.6 \mu \mathrm{m}$, indicates the existence of a photodissociation region (PDR) probably created by a nearby stellar source. Since PAHs molecules are destroyed inside the ionized gas of an HII region (Povich et al. 2007), but fluorescing when irradiated with weak ultraviolet radiation, they indicate the limits of the ionization front and delineate the boundaries of the bubble nebula, tracing the distribution of the parental molecular gas in which S169 is developing. This scenario is also supported by the presence of $\mathrm{H} \alpha$ emission at the center of the bubble nebula. A distinctive feature in the emission of S169 is the presence of two bright borders at the southern region of the IR nebula, which are discernible in Fig. 1 in yellow tonalities. The spatial distribution of the IR emission gives a good hint of the distribution of the molecular gas and the location of the densest regions, very likely associated with the two bright borders described before. As can be seen from Fig. 1, the IR emission at $8.0 \mu \mathrm{m}$ toward the west of the cavity is much weaker and composed of a faint arc-like filament approximately at $\mathrm{RA}=12^{\mathrm{h}} 35^{\mathrm{m}} 10^{\mathrm{s}}$.

The nebula S169 is placed near the IR source IRAS 123266245 , which has characteristics of a young stellar object (YSO), according to the photometric criteria from Junkes et al. (1992). Substantial evidence suggests that IRAS 12326-6245 is a dense molecular core where massive star formation is taking place, namely: (a) this source was observed in the $\operatorname{CS}(2-1)$ line at a velocity of $-39.4 \mathrm{~km} \mathrm{~s}^{-1}$ (Bronfman et al. 1996; Osterloh et al.
1997). It was also observed by Zinchenko et al. (2000) in several lines of HNCO at frequencies between 290 and $806 \mathrm{GHz}$. This indicates undoubtedly the existence of dense molecular gas associated with the source; (b) emission from dense dust has been previously detected at $1.2 \mathrm{~mm}$ as a compact source (Faúndez et al. 2004; Hill et al. 2005; Miettinen et al. 2006). The source is also a strong emitter at $870 \mu \mathrm{m}$ (Dedes et al. 2011; König et al. 2017; see Fig. 1); (c) $\mathrm{CH}_{3} \mathrm{OH}$ maser emission, a common phenomenon in massive star forming regions, was reported by Caswell (2009) at a velocity of $-39.8 \mathrm{~km} \mathrm{~s}^{-1}$. OH and $\mathrm{H}_{2} \mathrm{O}$ maser emission was also detected at the source (Caswell 1998; MacLeod et al. 1998); (d) a variety of complex organic molecules have been detected in the source (Araya et al. 2005; Dedes et al. 2011). These kinds of molecules are mostly formed in the surface of dust grains and later evaporate into the gas phase. This is characteristic of "hot molecular cores" (HMC; Kurtz et al. 2000), which are better known to be associated with early stages of massive star formation; (e) molecular line maps indicate the presence of a very powerful bipolar outflow at the center of the source (Henning et al. 2000).

In this work, we present a complete multifrequency analysis of the ISM around S169 using high spatial resolution molecular observations, which are necessary to establish the excitation conditions in the molecular environs, as well as to estimate their physical properties and to investigate the kinematics of the region. The analysis of the molecular observations are complemented with an analysis of a multifrequency archival dataset, which altogether contribute to our understanding of the impact of stellar feedback and how the ionized gas interacts with its molecular environment. We also investigate the status of the starformation processes in the region. In that context, the proximity between S169 and IRAS 12326-6245 is crucial for the analysis since it will allow us to better understand the high-mass 
Table 1. Observational parameters for the observed transitions.

\begin{tabular}{lcccr}
\hline \hline $\begin{array}{l}\text { Molecular } \\
\text { transition }\end{array}$ & $\begin{array}{c}\text { Frequency } \\
(\mathrm{GHz})\end{array}$ & $\begin{array}{c}\text { Beam } \\
\left({ }^{\prime \prime}\right)\end{array}$ & $\begin{array}{c}\text { Velocity } \\
\text { resolution } \\
\left(\mathrm{km} \mathrm{s}^{-1}\right)\end{array}$ & $\begin{array}{r}\text { rms } \\
\text { noise } \\
(\mathrm{K})\end{array}$ \\
\hline $\mathrm{CO}(2-1)$ & 230.538000 & $\sim 27$ & 0.3 & $\sim 0.3$ \\
${ }^{13} \mathrm{CO}(2-1)$ & 220.398677 & $\sim 28$ & 0.3 & $\sim 0.3$ \\
$\mathrm{C}^{18} \mathrm{O}(2-1)$ & 219.560357 & $\sim 28$ & 0.3 & $\sim 0.25$ \\
$\mathrm{HCN}^{(3-2)}$ & 265.886180 & $\sim 23$ & 0.3 & $\sim 0.15$ \\
$\mathrm{HCO}^{+}(3-2)$ & 267.557526 & $\sim 23$ & 0.3 & $\sim 0.15$ \\
\hline
\end{tabular}

star-formation process around IR dust bubbles. An analysis of dense molecular line tracers and submillimeter emission in IRAS 12326-6245 is also presented.

The source IRAS $12326-6245$ is believed to be placed at a distance of $4.4 \mathrm{kpc}$. Such distance was first estimated by Osterloh et al. (1997) and consistently adopted afterwards by several authors (e.g., Henning et al. 2000; Faúndez et al. 2004; Dedes et al. 2011). The distance to IRAS 12326-6245 is also matter of some debate in this work.

\section{Observations and databases}

\subsection{Molecular observations}

The molecular observations presented in this paper were made in December 2016 with the Atacama Pathfinder EXperiment ${ }^{1}$ (APEX) telescope (Güsten et al. 2006) at Llano de Chajnantor (Chilean Andes). As the front end for the observations, we used the APEX-1 receiver of the Swedish Heterodyne Facility Instrument (SHeFI; Vassilev et al. 2008). The back end for all observations was the eXtended bandwidth Fast Fourier Transform Spectrometer2 (XFFTS2), with a $2.5 \mathrm{GHz}$ bandwidth divided into 32768 channels. The observed transitions and basic observational parameters are summarized in Table 1. Calibration was done by the chopper-wheel technique and the output intensity scale given by the system is $T_{\mathrm{A}}$, which represents the antenna temperature corrected for atmospheric attenuation. The observed intensities were converted to the main-beam brightness temperature scale by $T_{\mathrm{mb}}=T_{\mathrm{A}} / \eta_{\mathrm{mb}}$, where $\eta_{\mathrm{mb}}$ is the main beam efficiency. For the APEX-1 receiver we adopted $\eta_{\mathrm{mb}}=0.75$.

Observations were made using the on-the-fly (OTF) mode with a step size of $10^{\prime \prime}$ (full sampling) in two orthogonal scan directions along RA and Dec (J2000). For the lines $\mathrm{CO}(2-1),{ }^{13} \mathrm{CO}(2-1)$, and $\mathrm{C}^{18} \mathrm{O}(2-1)$ the observed region was of $\sim 14^{\prime} \times 14^{\prime}$ in size centered on RA, Dec $(\mathrm{J} 2000)=\left(12^{\mathrm{h}} 35^{\mathrm{m}} 44^{\mathrm{s}}\right.$, $\left.-63^{\circ} 00^{\prime} 08^{\prime \prime}\right)$. For the lines $\mathrm{HCN}(3-2)$ and $\mathrm{HCO}^{+}(3-2)$ the observed region was $\sim 4^{\prime} \times 4^{\prime}$ in size centered on the position of the source IRAS $12326-6245$ at RA, Dec $(J 2000)=\left(12^{\mathrm{h}} 35^{\mathrm{m}} 33^{\mathrm{s}}\right.$, $\left.-63^{\circ} 02^{\prime} 56^{\prime \prime}\right)$. The data reduction was carried out with the CLASS90 package of the IRAM GILDAS software ${ }^{2}$.

\subsection{Archival data}

Infrared data. (a) Images of ATLASGAL at $870 \mu \mathrm{m}$ (345 GHz) (Schuller et al. 2009). This survey covers the

\footnotetext{
1 This publication is based on data acquired with the Atacama Pathfinder Experiment (APEX) under program ID C-098.F-9701. APEX is a collaboration between the Max-Planck-Institut fur Radioastronomie, the European Southern Observatory, and the Onsala Space Observatory.

2 http://wWw.iram.fr/IRAMFR/GILDAS
}

inner Galactic plane, $l=300^{\circ}$ to $60^{\circ},|b| \leq 1 .^{\circ} 5$, with the root mean square (rms) noise in the range of 0.05-0.07 Jy beam $^{-1}$. The beam size at $870 \mu \mathrm{m}$ is 19.2 . (b) Images from the $\mathrm{Herschel}^{3}$ Infrared GALactic (Hi-GAL) plane survey key program (Molinari et al. 2010). We used images from the photometric array camera and spectrometer (PACS) survey at 70 and $100 \mu \mathrm{m}$, with FWHM of $5{ }^{\prime \prime} 5$ and $11^{\prime \prime}$, respectively, and from the spectral and photometric imaging receiver (SPIRE) at $350 \mu \mathrm{m}$ with a FHWM of 25". (c) Images from Spitzer at 5.8 and $8.0 \mu \mathrm{m}$ from the Galactic Legacy Infrared Mid-Plane Survey Extraordinaire (Spitzer-GLIMPSE, Benjamin et al. 2003), retrieved from the Spitzer Science Center ${ }^{4}$. The images have a spatial resolution of $\sim 2^{\prime \prime}$.

Radio continuum data. Mosaics obtained from the Sydney University Molonglo Sky Survey ${ }^{5}$ (SUMSS; Bock et al. 1999). This widefield radio imaging survey covers the southern sky at $843 \mathrm{MHz}$. The resolution is $43^{\prime \prime} \times 43^{\prime \prime} \operatorname{cosec}(\delta)$ and the rms noise level is $\sim 1 \mathrm{mJy}^{\text {beam }^{-1}}$.

HI data. Data cubes from the Southern Galactic Plane Survey (SGPS; McClure-Griffiths et al. 2005). This survey includes the regions limited by Galactic longitude $253^{\circ} \leq l \leq$ $358^{\circ}$ (SGPS-1) and $5^{\circ} \leq l \leq 20^{\circ}$ (SGPS-2) and Galactic latitude $|b| \leq 1$.5. Interferometric observations obtained with the Australia Telescope Compact Array (ATCA) are combined with single dish data from the $64 \mathrm{~m}$ Parkes radiotelescope. The final products have an angular resolution of $\sim 2^{\prime}$ and a rms sensitivity

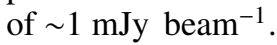

Narrow band $\mathrm{H} \alpha$ data. Retrieved from the SuperCOSMOS H $\alpha$ Survey $^{6}$ (SHS). The images have a spatial resolution of $\sim 1^{\prime \prime}$ (Parker et al. 2005).

To investigate ionizing stars candidates and YSO candidates in the region of the nebula, we made use of the IRAS Point Source Catalog ${ }^{7}$ (Beichman et al. 1988), the MSX Infrared Point Source Catalog 8 (Egan et al. 2003), the WISE All-Sky Source Catalog 9 (Wright et al. 2010), the GLIMPSE point source cata$\log ^{10}$ (Benjamin et al. 2003), the Two Micron All Sky Survey $(2 \mathrm{MASS})^{11}$ (Skrutskie et al. 2006), the American Association of Variable Star Observers (AAVSO) Photometric All-Sky SurveyA (APASS) ${ }^{12}$ (Henden et al. 2010), the ASCC-2.5 V3: All-sky Compiled Catalog of 2.5 million stars (Kharchenko \& Roeser 2009), and the Astrometric catalog GAIA Data Release $2^{13}$ (DR2; Gaia Collaboration 2016, 2018).

\section{Molecular and dust emission}

\subsection{Emission of $\mathrm{CO}$ and its isotopologs}

Figure 2 portrays the total averaged spectra of $\mathrm{CO}(2-1)$, ${ }^{13} \mathrm{CO}(2-1)$, and $\mathrm{C}^{18} \mathrm{O}(2-1)$ in a region of $\sim 14^{\prime} \times 14^{\prime}$ in a

\footnotetext{
3 Herschel is an ESA space observatory with science instruments provided by European-led Principal Investigator consortia and with important participation from NASA (http://www . cosmos . esa. int/ web/herschel/science-archive).

4 https://ssc.spitzer.caltech.edu/

5 http://www.astrop.physics.usyd.edu.au/mosaics

6 http://www-wfau.roe.ac.uk/sss/halpha/hapixel.html

7 https://irsa.ipac.caltech.edu/Missions/iras.html

8 https://irsa.ipac.caltech.edu/Missions/msx.html

9 https://irsa.ipac.caltech.edu/Missions/wise.html

${ }^{10}$ https://irsa.ipac.caltech.edu/Missions/spitzer.html

${ }^{11}$ https://irsa.ipac.caltech.edu/Missions/2mass.html

12 https://www. aavso.org/apass

${ }^{13}$ https://www. cosmos. esa.int/web/gaia/home
} 


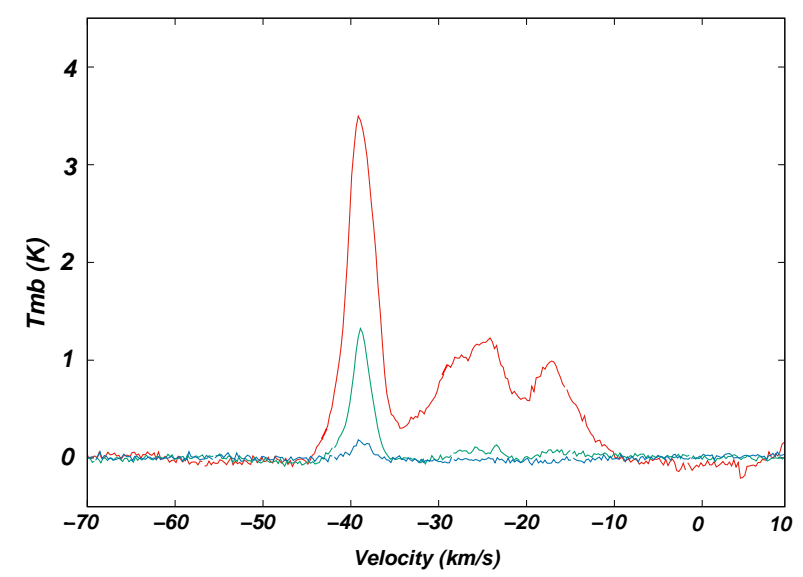

Fig. 2. Total averaged spectra of $\mathrm{CO}(2-1)($ red $),{ }^{13} \mathrm{CO}(2-1)$ (green), and $\mathrm{C}^{18} \mathrm{O}(2-1)$ (blue) obtained within a region of $\sim 14^{\prime} \times 14^{\prime}$ centered on RA, Dec $(\mathrm{J} 2000)=\left(12^{\mathrm{h}} 35^{\mathrm{m}} 44^{\mathrm{s}},-63^{\circ} 00^{\prime} 08^{\prime \prime}\right)$.

position that is approximately at the center of the bubble nebula. The CO emission clearly depicts a strong narrow velocity component peaking at approximately $-39 \mathrm{~km} \mathrm{~s}^{-1}$ and two weaker broad velocity components peaking at approximately $-25 \mathrm{~km} \mathrm{~s}^{-1}$ and approximately $-17 \mathrm{~km} \mathrm{~s}^{-1}$. For the sake of the analysis, these components will be hereafter referred to as component $\mathrm{A}$, component $\mathrm{B}$, and component $\mathrm{C}$, respectively.

The peak velocity of component A, which is very likely dominated by the emission of IRAS 12326-6245, is almost coincident with the peak velocity of reported non-masing and masing emission lines towards the IRAS source (Bronfman et al. 1996; Zinchenko et al. 2000; Henning et al. 2000; Dedes et al. 2011; Araya et al. 2005; Caswell 1998, 2009; MacLeod et al. 1998). This component is also detected in ${ }^{13} \mathrm{CO}$ and $\mathrm{C}^{18} \mathrm{O}$ emissions, which indicates that it is composed of high density molecular gas. Components $\mathrm{B}$ and $\mathrm{C}$, on the other hand, are barely detected in the ${ }^{13} \mathrm{CO}$ emission and not detected in the $\mathrm{C}^{18} \mathrm{O}$ emission. As opposed to the case of the velocity of component A, neither masing nor non-masing emission lines were reported for IRAS $12326-6245$ at the velocity of components B and C.

\subsection{Component $A$}

In Fig. 3, we show the spatial distribution of the $\mathrm{CO}(2-1)$, ${ }^{13} \mathrm{CO}(2-1)$, and $\mathrm{C}^{18} \mathrm{O}(2-1)$ lines in the velocity interval from approximately -42 to $-37 \mathrm{~km} \mathrm{~s}^{-1}$. As expected according to the IR emission distribution (see Fig. 1), the bulk of the molecular emission appears concentrated toward the eastern and southern borders of the IR nebula, with no significant molecular emission detected toward the western and northeastern regions. The morphology and location of component A, $\mathrm{H} \alpha$ emission (see Fig. 1), and radio continuum emission (see Sect. 4) suggest that the ionized gas is expanding against the molecular cloud in the eastern and southern borders. The molecular emission at the southern border of the bubble seems to be tracing the densest areas, which are probably sculpted by the action of the HII region. The morphology and location of component A with respect to the near IR emission is similar to many other in Galactic IR bubbles found in the literature (e.g., Zavagno et al. 2006; Deharveng et al. 2008, 2009; Anderson et al. 2015; Liu et al. 2015; Cappa et al. 2016; Duronea et al. 2017; Devine et al. 2018). For a better visualization and comparison with the IR emission, in panel a of Fig. 4 we show the emission of ${ }^{13} \mathrm{CO}(2-1)$ of component A superimposed on the IRAC-GLIMPSE emissions at 8.0 and $5.8 \mu \mathrm{m}$.
From Fig. 3, it can also be seen that the $\mathrm{CO}$ emission of component $\mathrm{A}$ is more extended than that of ${ }^{13} \mathrm{CO}$ and $\mathrm{C}^{18} \mathrm{O}$. This indicates that ${ }^{13} \mathrm{CO}$ and $\mathrm{C}^{18} \mathrm{O}$ lines, optically thinner than that of $\mathrm{CO}$ (see below), are actually tracing the distribution of the densest molecular gas surrounding the bubble nebula. In order to perform a more detailed analysis of the dense molecular gas in component $\mathrm{A}$, we have roughly identified six molecular condensations in the ${ }^{13} \mathrm{CO}(2-1)$ emission using the $\mathrm{C}^{18} \mathrm{O}(2-1)$ emission (which traces the denser gas) as a reference. The identification was done using the $1.5 \mathrm{~K} \mathrm{~km} \mathrm{~s}^{-1}$ contour level in the ${ }^{13} \mathrm{CO}(2-1)$ emission. The condensations are labeled in Fig. 3 as MC1, MC2, MC3, MC4, MC5, and MC6. These condensations are the places where star formation is likely to be taking place (see Sect. 5). In panels b to i of Fig. 4, we show the channel maps of ${ }^{13} \mathrm{CO}$ in the velocity range from -36.7 to $-42.5 \mathrm{~km} \mathrm{~s}^{-1}$ in intervals of $0.7 \mathrm{~km} \mathrm{~s}^{-1}$. In order to compare the molecular emission distribution with the warm and cold dust emission, the ${ }^{13} \mathrm{CO}$ emission was superimposed on the 8.0 and $5.8 \mu \mathrm{m}$ (IRACGLIMPSE) and the $870 \mu \mathrm{m}$ (ATLASGAL) maps. Figure 4 shows that MC3, located at RA, Dec $(J 2000)=\left(12^{\mathrm{h}} 35^{\mathrm{m}} 35^{\mathrm{s}}\right.$, $\left.-63^{\circ} 02^{\prime} 25^{\prime \prime}\right)$, is the only molecular condensation that is present in the entire velocity range. The position and size of MC3 perfectly match with those of the bright spot seen at $870 \mu \mathrm{m}$. This condensation certainly represents the carbon monoxide counterpart of IRAS 12326-6245, which reveals the physical association between S169 and the IRAS source. In the velocity interval from -36.7 to $-38.2 \mathrm{~km} \mathrm{~s}^{-1}$ condensation MC1 becomes noticeable, reaching its peak temperature in the velocity interval from -38.9 to $-39.6 \mathrm{~km} \mathrm{~s}^{-1}$, close to a $8.0 \mu \mathrm{m}$ source seen at RA, Dec $(\mathrm{J} 2000)=\left(12^{\mathrm{h}} 35^{\mathrm{m}} 24^{\mathrm{s}},-63^{\circ} 05^{\prime} 42^{\prime \prime}\right)$. It can be detected till a velocity of $-40.4 \mathrm{~km} \mathrm{~s}^{-1}$. For the case of condensation MC2, it can be first noticed in the velocity interval from -36.7 to $-38.9 \mathrm{~km} \mathrm{~s}^{-1}$, where its emission merges with MC1. Its peak emission is observed in the velocity range from -37.4 to $-38.2 \mathrm{~km} \mathrm{~s}^{-1}$ and is coincident with another $8.0 \mu \mathrm{m}$ source seen at RA, Dec $(\mathrm{J} 2000)=\left(12^{\mathrm{h}} 35^{\mathrm{m}} 29^{\mathrm{s}},-63^{\circ} 04^{\prime} 25^{\prime \prime}\right)$. Condensation MC4 becomes detectable in the velocity interval from -36.7 to $-37.4 \mathrm{~km} \mathrm{~s}^{-1}$, reaching to its maximum peak emission in the velocity interval from -38.2 to $-38.9 \mathrm{~km} \mathrm{~s}^{-1}$. It can be noticed till a velocity of $-39.6 \mathrm{~km} \mathrm{~s}^{-1}$ although its emission appears blended with MC2. Regarding condensations MC5 and MC6, they become detectable in the velocity range from -36.7 to $-37.4 \mathrm{~km} \mathrm{~s}^{-1}$. Their emission distribution is quite irregular and achieve their peak temperatures in the velocity range from -38.2 to $-38.9 \mathrm{~km} \mathrm{~s}^{-1}$. Condensation MC4 is detectable till a velocity of $-39.6 \mathrm{~km} \mathrm{~s}^{-1}$, while MC5 till a velocity of $-40.4 \mathrm{~km} \mathrm{~s}^{-1}$.

Figure 4 also shows a strong morphological correlation between the brightest parts of the PDR (described in Sect. 1) and the molecular condensations MC3, MC4, and MC5, especially at velocities between -38.2 and $-38.9 \mathrm{~km} \mathrm{~s}^{-1}$. In particular, it is noticeable how the bright emission feature detected at 5.8 and $8.0 \mu \mathrm{m}$ borders the area of MC3, which are likely to be exposed to stellar radiation.

To estimate the physical properties of the ${ }^{13} \mathrm{CO}$ condensations, we obtained six ${ }^{13} \mathrm{CO}$ emission maps (not shown here) integrated in the velocity interval in which each condensation is detected. The deconvolved effective radius of the condensations derived from the ${ }^{13} \mathrm{CO}$ line, $R_{\mathrm{D}}^{13} \mathrm{CO}$, is calculated as:

$R_{\mathrm{D}}^{13} \mathrm{CO}=\sqrt{R_{\mathrm{eff}}^{2}-(H P B W / 2)^{2}}$,

where $R_{\mathrm{eff}}$ is the effective radii of the condensation $\left(R_{\text {eff }}=\sqrt{A_{\text {cond }} / \pi}\right.$ ), being $A_{\text {cond }}$ the area of the condensation, and HPBW is the half-power beam width of the instrument. 

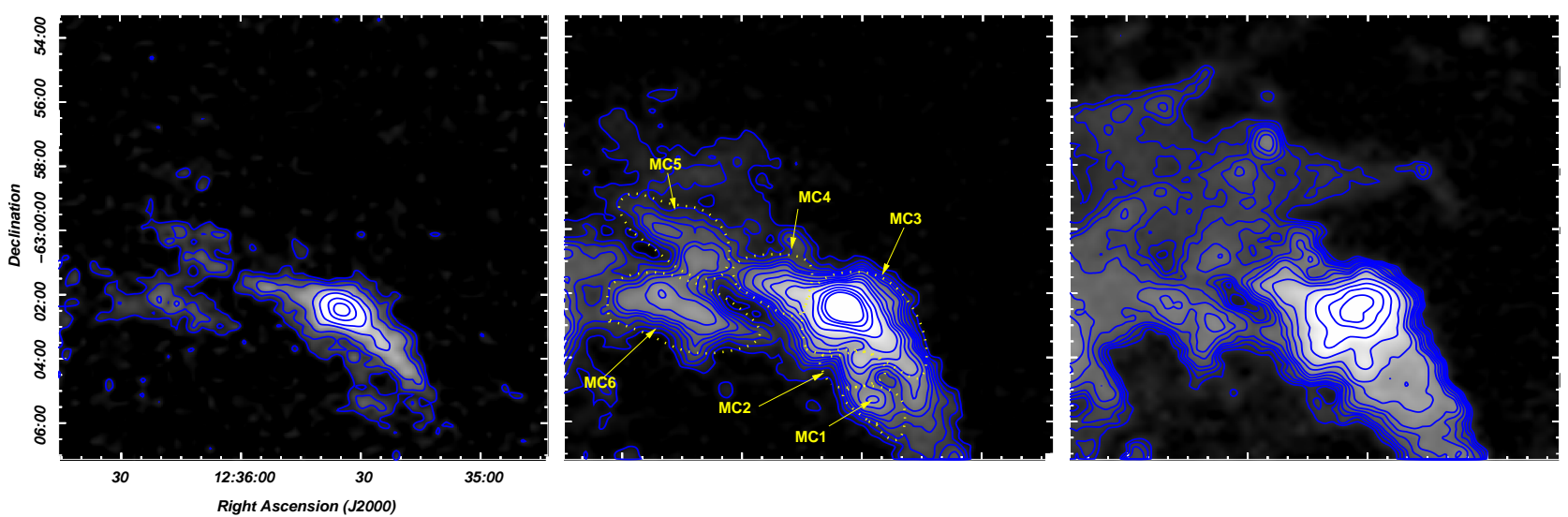

Fig. 3. Spatial distribution of the $\mathrm{C}^{18} \mathrm{O}(2-1)$ emission (left panel), ${ }^{13} \mathrm{CO}(2-1)$ emission (middle panel), and $\mathrm{CO}(2-1)$ emission (right panel) in the velocity interval from approximately -42 to $-37 \mathrm{~km} \mathrm{~s}^{-1}$ (component A). In the left panel, the contours are $0.35(\sim 3 \mathrm{rms}), 0.7,1.15,2,3,5$, and $7 \mathrm{~K} \mathrm{~km} \mathrm{~s}^{-1}$. In the middle panel the contour levels are 0.5 ( $\left.\sim 6 \mathrm{rms}\right), 1,1.5,2,2.5,3,4,5,7,9,11,13$, and $15 \mathrm{~K} \mathrm{~km} \mathrm{~s}^{-1}$. In the right panel the contour levels are $1.5(\sim 20 \mathrm{rms}), 2,2.5,3,4,5,6,8,10,14,18,22$, and $26 \mathrm{~K} \mathrm{~km} \mathrm{~s}^{-1}\left(T_{\mathrm{mb}}\right)$. Identified molecular condensations are indicated in yellow over the ${ }^{13} \mathrm{CO}(2-1)$ emission.

To estimate $R_{\mathrm{D}}^{13} \mathrm{CO}$ and other parameters, we adopted a distance of $2.03_{-0.61}^{+0.77} \mathrm{kpc}$ (see Sect. 6.2). Then we obtained the effective radii of $0.40,0.27,0.94,0.49,0.75$, and $0.86 \mathrm{pc}$ for $\mathrm{MC} 1, \mathrm{MC} 2$, MC3, MC4, MC5, and MC6, respectively.

Assuming that all rotational levels are thermalized with the same excitation temperature (LTE) and that the emission is optically thick, we derived the excitation temperature of each molecular condensation, $T_{\text {exc }}$, from the $\mathrm{CO}(2-1)$ line using

$T_{\text {peak }}=T_{12}^{*}\left[\left(e^{\frac{T_{12}^{*}}{T_{\mathrm{exc}}}}-1\right)^{-1}-\left(e^{\frac{T_{12}^{*}}{T_{\mathrm{bg}}}}-1\right)^{-1}\right]$

where $T_{12}^{*}=h v_{12} / k$, being $v_{12}$ the frequency of the ${ }^{12} \mathrm{CO}(2-1)$ line, and $T_{\mathrm{bg}}=2.7 \mathrm{~K}$. To obtain the peak main beam temperature of the $\mathrm{CO}(2-1),{ }^{13} \mathrm{CO}(2-1)$, and $\mathrm{C}^{18} \mathrm{O}(2-1)$ lines $\left(T_{\text {peak }}\right)$, we used Gaussian fits over the spectra profiles obtained integrating in the direction of the peak emission of the ${ }^{13} \mathrm{CO}$ condensations (in all the cases coincident with those of the $\mathrm{C}^{18} \mathrm{O}$ emission) over an area equivalent to the area of the beam. It is worth to point out that a single Gaussian component was considered for MC3, which shows self-absorption in the spectrum of $\mathrm{CO}(2-1)$ at the velocity of the peak emission of ${ }^{13} \mathrm{CO}(2-1)$ and $\mathrm{C}^{18} \mathrm{O}(2-1)$ (Henning et al. 2000; Dedes et al. 2011). Excitation temperatures derived with Eq. (2) are then $21.3 \mathrm{~K}, 33.8 \mathrm{~K}, 47.8 \mathrm{~K}, 21.2 \mathrm{~K}$, and 16.0 K, and 16.8 K for MC1, MC2, MC3, MC4, MC5, and MC6, respectively.

The optical depths $(\tau)$ of the ${ }^{13} \mathrm{CO}(2-1)$ and $\mathrm{C}^{18} \mathrm{O}(2-1)$ lines, denoted in the next equations as $\tau^{13}$ and $\tau^{18}$, respectively, were obtained assuming that the excitation temperature is the same for $\mathrm{CO}(2-1),{ }^{13} \mathrm{CO}(2-1)$, and $\mathrm{C}^{18} \mathrm{O}(2-1)$, and using the expressions

$\tau^{13}=-\ln \left[1-\frac{T_{\mathrm{peak}}^{13} \mathrm{CO}}{T_{13}^{*}}\left[\left(e^{\frac{T_{13}^{*}}{T_{\mathrm{exc}}}}-1\right)^{-1}-\left(e^{\frac{T_{13}^{*}}{T_{\mathrm{bg}}}}-1\right)^{-1}\right]^{-1}\right]$

and

$\tau^{18}=-\ln \left[1-\frac{T_{\text {peak }} \mathrm{C}^{18} \mathrm{O}}{T_{18}^{*}}\left[\left(e^{\frac{T_{18}^{*}}{T_{\mathrm{exc}}}}-1\right)^{-1}-\left(e^{\frac{T_{18}^{*}}{T_{\mathrm{bg}}}}-1\right)^{-1}\right]^{-1}\right]$, where $T_{13}^{*}=h v_{13} / k, T_{18}^{*}=h v_{18} / k$, with $v_{13}$ and $v_{18}$ the frequencies of the ${ }^{13} \mathrm{CO}(2-1)$ and $\mathrm{C}^{18} \mathrm{O}(2-1)$ lines, respectively. We can also estimate the optical depth of the $\mathrm{CO}(2-1)$ line from the ${ }^{13} \mathrm{CO}(2-1)$ line using

$\tau^{12}=\left[\frac{v^{13}}{v^{12}}\right]^{2} \times\left[\frac{\Delta v^{13}}{\Delta v^{12}}\right] \times\left[\frac{\mathrm{CO}}{{ }^{13} \mathrm{CO}}\right] \tau^{13}$,

where $\mathrm{CO} /{ }^{13} \mathrm{CO}$ is the isotope ratio (assumed to be $\sim 62$; Langer \& Penzias 1993); $\Delta v^{13}$ and $\Delta v^{12}$ are defined as the full width half maximum (FWHM) of the spectra of the ${ }^{13} \mathrm{CO}$ and $\mathrm{CO}$ lines, respectively, which are derived by using a single Gaussian fitting $\left(F W H M=2 \times \sqrt{2 \ln 2} \times \sigma_{\text {gauss }}\right)$.

In LTE, the ${ }^{13} \mathrm{CO}$ and $\mathrm{C}^{18} \mathrm{O}$ column densities can be estimated from the ${ }^{13} \mathrm{CO}(2-1)$ and $\mathrm{C}^{18} \mathrm{O}(2-1)$ line using

$N\left({ }^{13} \mathrm{CO}\right)=3.23 \times 10^{14}\left[\frac{e^{\frac{T_{13}^{*}}{T_{\mathrm{exc}}}}}{1-e^{-\frac{T_{13}^{*}}{T_{\mathrm{exc}}}}}\right] T_{\mathrm{exc}} \int \tau^{13} \mathrm{~d} v\left(\mathrm{~cm}^{-2}\right)$

and

$N\left(\mathrm{C}^{18} \mathrm{O}\right)=3.21 \times 10^{14}\left[\frac{e^{\frac{T_{18}^{*}}{T_{\mathrm{exc}}}}}{1-e^{-\frac{T_{18}^{*}}{T_{\mathrm{exc}}}}}\right] T_{\mathrm{exc}} \int \tau^{18} \mathrm{~d} v\left(\mathrm{~cm}^{-2}\right)$.

The integral in Eqs. (6) and (7) can be approximated by

$T_{\mathrm{exc}} \int \tau \mathrm{d} v \approx \frac{\tau}{1-e^{(-\tau)}} \int T_{\mathrm{mb}} \mathrm{dv}$

This approximation helps to eliminate to some extent optical depth effects and is good within $15 \%$ for $\tau<2$ (Rohlfs \& Wilson 2004). Bearing in mind the values of $\tau^{13}$ obtained, the approximation is appropriate for our region. Estimations of the $\mathrm{H}_{2}$ column density, $N\left(\mathrm{H}_{2}\right)$, were obtained from both the ${ }^{13} \mathrm{CO}$ and $\mathrm{C}^{18} \mathrm{O}$ column densities, and adopting abundances $X\left[{ }^{13} \mathrm{CO}\right]=7.1 \times 10^{5}$ and $X\left[\mathrm{C}^{18} \mathrm{O}\right]=5.9 \times 10^{6}$ (Frerking et al. 1982). For the optical depth and column density, we estimated uncertainties of about 20 and $25 \%$, respectively, arising mostly from calibration uncertainties of about 20\% (Dumke \& Mac-Auliffe 2010).

The total hydrogen mass of each clump was calculated using

$M\left(\mathrm{H}_{2}\right)=\left(m_{\text {sun }}\right)^{-1} \mu m_{\mathrm{H}} \Omega N\left(\mathrm{H}_{2}\right) d^{2} \quad\left(M_{\odot}\right)$, 

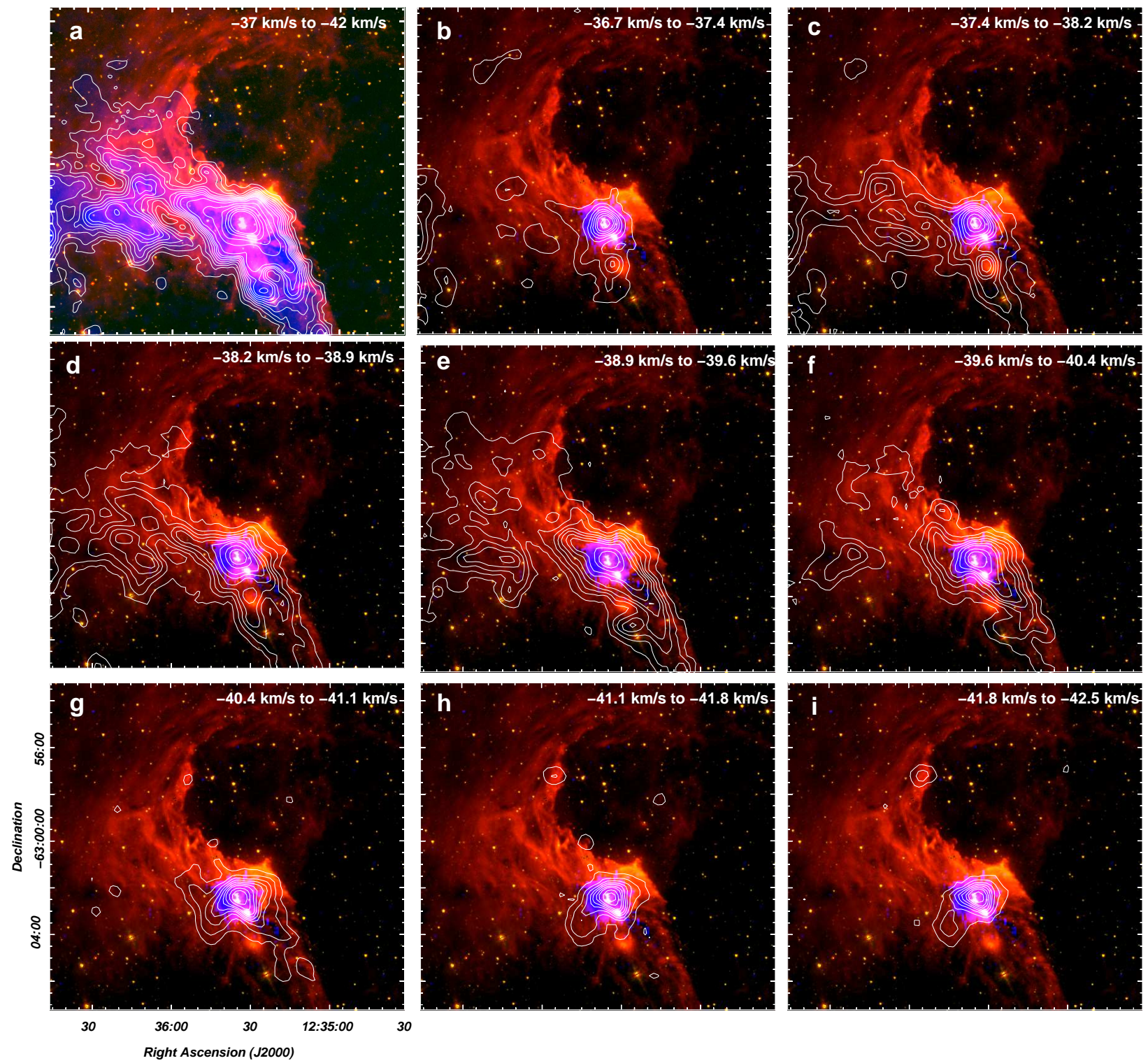

Fig. 4. Panel a: emission distribution of the ${ }^{13} \mathrm{CO}(2-1)$ line in the total velocity range from -37 to $-42 \mathrm{~km} \mathrm{~s}^{-1}$ (white contours and blue color scale) superimposed on the IRAC-GLIMPSE images at 8.0 and $5.8 \mu \mathrm{m}$ (red and green color scales, respectively). The contours levels are the same than those shown in the middle panel of Fig. 3. Panels $b$ to $i$ : channel maps of the ${ }^{13} \mathrm{CO}(2-1)$ line emission in velocity intervals of $0.7 \mathrm{~km} \mathrm{~s}{ }^{-1}$ (white contours) in the velocity range from -36.7 to $-42.5 \mathrm{~km} \mathrm{~s}^{-1}$ superimposed on the 8.0 and $5.8 \mu \mathrm{m}$ (IRAC-GLIMPSE) emissions (red and green color scales) and $870 \mu$ m ATLASGAL emission (blue color scale) inside the $3 \mathrm{rms}$ limit. Contours levels are $0.9(\sim 5.5 \mathrm{rms}), 2.6,4.4,6.2,7.9,10,12,16$, and $20 \mathrm{~K} \mathrm{~km} \mathrm{~s}^{-1}$. The velocity interval is indicated in the top right corner of each panel.

where $m_{\text {sun }}$ is the solar mass $\left(\sim 2 \times 10^{33} \mathrm{~g}\right), \mu$ is the mean molecular weight, which is assumed to be equal to 2.76 after allowing for a relative helium abundance of $25 \%$ by mass (Yamaguchi et al. 1999), $m_{\mathrm{H}}$ is the hydrogen atom mass $\left(\sim 1.67 \times 10^{-24} \mathrm{~g}\right), \Omega$ is the solid angle of the ${ }^{13} \mathrm{CO}$ emission expressed in sr, $d$ is the adopted distance expressed in $\mathrm{cm}$, and $N\left(\mathrm{H}_{2}\right)$ is the $\mathrm{H}_{2}$ column density obtained from $N\left({ }^{13} \mathrm{CO}\right)$. Uncertainties in molecular masses are about $60 \%$, and originate mainly from a distance uncertainty of about $30 \%$ (see Sect. 6.2). The derived physical parameters are presented in Table 2.

In order to perform a study of the molecular gas in IRAS 12326-6245, we also present an analysis for MC3 using the high-density tracer lines $\mathrm{HCN}(3-2)$ and $\mathrm{HCO}^{+}(3-2)$. In Fig. 5, we show the emission of these lines in the velocity intervals from -47.6 to $-29.2 \mathrm{~km} \mathrm{~s}^{-1}$ and -44.8 to $-32.5 \mathrm{~km} \mathrm{~s}^{-1}$, respectively. Their emissions show a good morphological correspondence with that at $870 \mu \mathrm{m}$ (see Fig. 4) and seem to be delineating an obscured region in the 8.0 and $5.8 \mu \mathrm{m}$ emissions. They are certainly the $\mathrm{HCN}$ and $\mathrm{HCO}^{+}$counterparts of the molecular condensation MC3. In order to estimate the $\mathrm{HCN}$ and $\mathrm{HCO}^{+}$column densities, we used LTE models numerically approximated with the Markov chain Monte Carlo (MCMC) method (Foreman-Mackey et al. 2013). The lines in LTE were simulated and compared with the observations (e.g., Comito et al. 2005; Möller et al. 2017). The solutions presented were obtained from the averaged spectra considering different offsets since the individual solutions for each one of them were similar. That is a consequence of the homogeneous emission traced by the species analyzed here. In Fig. 5, we show the average spectra (in antenna temperature) of $\mathrm{HCN}(3-2)$ and 
Table 2. Observed parameters obtained from the emission lines and physical properties derived for the molecular condensations MC1 to MC6.

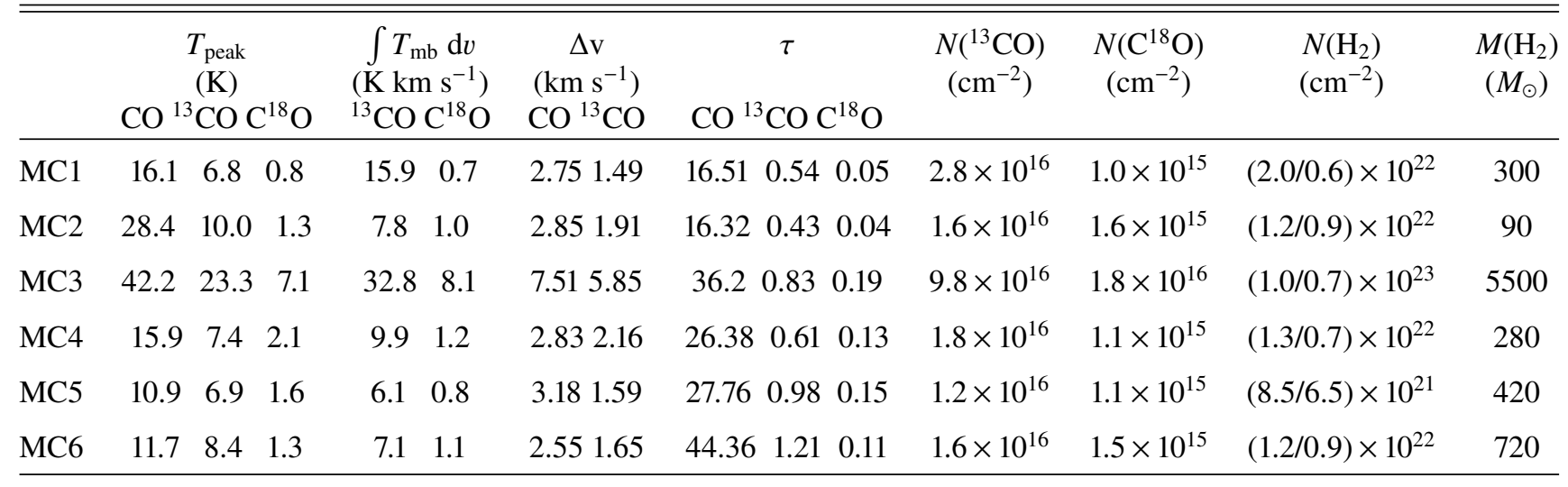

$\mathrm{HCO}^{+}$(3-2). The results presented here were obtained by taking into account those reported in IRAS $12326-6245$ by Dedes et al. (2011), who assumed temperature values of 150 and $50 \mathrm{~K}$ for the gas conditions of species in an apparent hot core region and extended envelope, respectively. For the sake of the analysis, we also included the optically thin lines of ${ }^{13} \mathrm{CO}(2-1)$ and $\mathrm{C}^{18} \mathrm{O}(2-1)$. Then, assuming an excitation temperature of $T_{\text {exc }}=50 \mathrm{~K}$ for the $\mathrm{HCO}^{+}(3-2)$ line, we obtained a column density of $N\left(\mathrm{HCO}^{+}\right)=(5.2 \pm 0.1) \times 10^{13} \mathrm{~cm}^{-2}$. For the ${ }^{13} \mathrm{CO}(2-1)$ and $\mathrm{C}^{18} \mathrm{O}(2-1)$ lines, also adopting $T_{\text {exc }}=50 \mathrm{~K}$, we obtained $N\left({ }^{13} \mathrm{CO}\right)=(2.01 \pm 0.04) \times 10^{17} \mathrm{~cm}^{-2}$ and $N\left(\mathrm{C}^{18} \mathrm{O}\right)=(3.9 \pm 0.1) \times 10^{16} \mathrm{~cm}^{-2}$. For the case of the HCN (3-2) line, LTE models suggest a physical condition related with the hot core region. Based on the hypothesis stating that $T_{\text {exc }}=150 \mathrm{~K}$, we derived $N(\mathrm{HCN})=(1.9 \pm 0.5) \times 10^{14} \mathrm{~cm}^{-2}$. Concerning the uncertainties, the results presented here are approximations that should be considered (at least) within the calibration uncertainty since several assumptions were adopted for single transitions. In order to inspect the quality of the fits, in Fig. 5 we also exhibit the residuals after subtracting the emission lines from their LTE models.

\subsection{Components $B$ and $C$}

As mentioned in Sect. 3.1, two weaker broad velocity molecular components peaking approximately at $-25 \mathrm{~km} \mathrm{~s}^{-1}$ (component B) and $-17 \mathrm{~km} \mathrm{~s}^{-1}$ (component C) were also detected in S169. These components are hardly detected in the ${ }^{13} \mathrm{CO}$ emission and not detected at all in the $\mathrm{C}^{18} \mathrm{O}$ emission, which certainly indicates that they are mostly composed by low-density gas. In order to analyze their spatial distribution, we constructed two CO emission maps integrated in the velocity intervals from -28.9 to $-18.9 \mathrm{~km} \mathrm{~s}^{-1}$ (component B) and from -18.2 to $-12.7 \mathrm{~km} \mathrm{~s}^{-1}$ (component $\mathrm{C}$ ). They are shown in Fig. 6 superimposed onto the 8.0 and $5.8 \mu \mathrm{m}$ emissions. For the case of component B, three molecular features are seen projected towards different regions of the nebula. The brightest feature (hereafter, feature 1) is seen approximately centered at RA, Dec $(\mathrm{J} 2000)=\left(12^{\mathrm{h}} 36^{\mathrm{m}} 34^{\mathrm{s}}\right.$, $\left.-62^{\circ} 55^{\prime} 13^{\prime \prime}\right)$ perfectly delineating the northeastern and northern borders of the IR nebula. As can be seen from the figure, the brightest regions of the molecular gas appear projected onto the faintest regions of the IR emission, which suggests that a PDR was formed over the surface of feature 1 (viewed from the side). Another feature is seen centered approximately at $\mathrm{RA}, \operatorname{Dec}(\mathrm{J} 2000)=\left(12^{\mathrm{h}} 36^{\mathrm{m}} 40^{\mathrm{s}},-63^{\circ} 06^{\prime} 00^{\prime \prime}\right)$ (feature 2). This feature appears bordering the southeastern edge of the nebula,
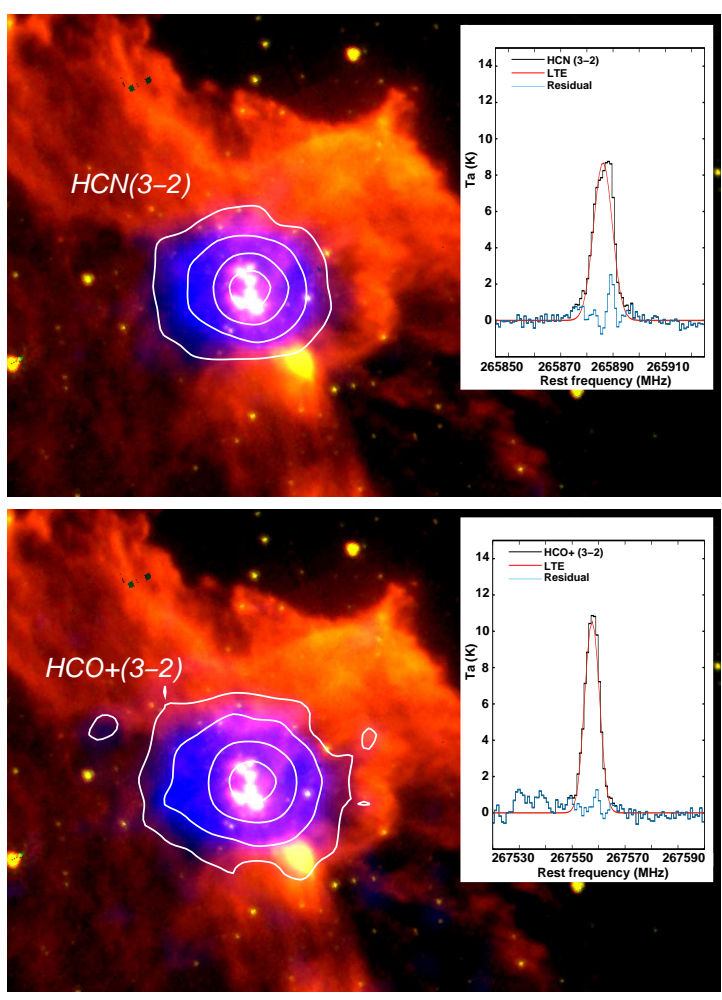

Fig. 5. Upper panel: spectral line and LTE model of the HCN (3-2) line obtained from LTE-MCMC calculations assuming $T_{\text {exc }}=150 \mathrm{~K}$ (see text), with a resulting density of $N=(1.9 \pm 0.5) \times 10^{14} \mathrm{~cm}^{-2}$. The blue line indicates the residual obtained from the subtraction between the observation and LTE model. In colors: spatial distribution of the $\mathrm{HCN}(3-2)$ emission in the velocity interval from -47.6 to $-29.2 \mathrm{~km} \mathrm{~s}^{-1}$ (color blue and white contours) superimposed on the IRAC 8 and $5.8 \mu \mathrm{m}$ emissions (red and green colors). Lower panel: same as above for the $\mathrm{HCO}^{+}(3-2)$ line, assuming $T_{\text {exc }}=50 \mathrm{~K}$, with a resulting density of $N=(5.2 \pm 0.1) \times 10^{13} \mathrm{~cm}^{-2}$. The $\mathrm{HCO}^{+}(3-2)$ emission distribution is in the velocity interval from -44.8 to $-32.5 \mathrm{~km} \mathrm{~s}^{-1}$. The contour levels for the $\mathrm{HCN}(3-2)$ are 0.25 ( $\sim 5 \mathrm{rms}), 0.85,2.2$, and $4.0 \mathrm{~K} \mathrm{~km} \mathrm{~s}^{-1}$; and for the $\mathrm{HCO}^{+}(3-2)$ line, they are $0.28(\sim 5 \mathrm{rms}), 0.8,2.4$, and $5 \mathrm{~K} \mathrm{~km} \mathrm{~s}^{-1}$

also projected onto the faintest regions of the IR nebula. The third feature is seen projected along the center of the nebula approximately at Dec $=-62^{\circ} 58^{\prime} 00^{\prime \prime}$ and is still noticeable at more negative velocities. Since this feature shows no morphological correspondence with any region of the IR nebula, a 

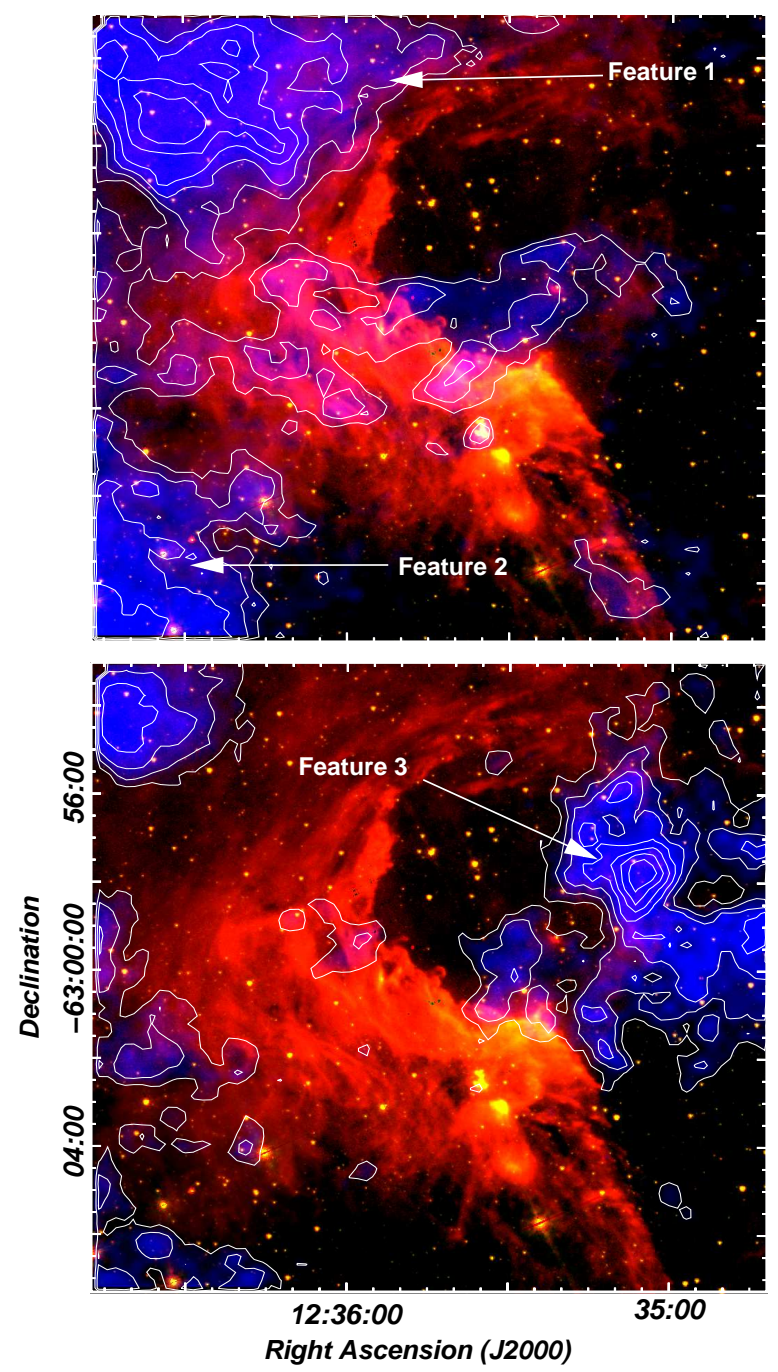

Fig. 6. Upper panel: spatial distribution of the $\mathrm{CO}$ emission (blue color scale and white contours) in the velocity interval from $-28.9 \mathrm{~km} \mathrm{~s}^{-1}$ to $-18.9 \mathrm{~km} \mathrm{~s}^{-1}$ (component B) superimposed on the IRAC $8 \mu \mathrm{m}$ and $5.8 \mu \mathrm{m}$ emissions (red and green color scales). The contour levels start from $0.95 \mathrm{~K} \mathrm{~km} \mathrm{~s}^{-1}(\sim 9 \mathrm{rms})$ in steps of $0.5 \mathrm{~K} \mathrm{~km} \mathrm{~s}^{-1}$. Lower panel: spatial distribution of the $\mathrm{CO}$ emission in the velocity interval from -18.2 to $-12.7 \mathrm{~km} \mathrm{~s}^{-1}$ (component $\mathrm{C}$ ). The contour levels start from $0.85 \mathrm{~K} \mathrm{~km} \mathrm{~s}^{-1}(\sim 7 \mathrm{rms})$ in steps of $0.4 \mathrm{~K} \mathrm{~km} \mathrm{~s}^{-1}$.

physical link with S169 cannot be suggested. Probably, it is an unconnected molecular structure located behind the nebula since no absorption features can be noticed along the brightest regions of the IR emission. Regarding component $\mathrm{C}$, only one molecular structure (feature 3 ) stands out, peaking approximately at RA, Dec $(\mathrm{J} 2000)=\left(12^{\mathrm{h}} 35^{\mathrm{m}} 05^{\mathrm{s}},-62^{\circ} 58^{\prime} 00^{\prime \prime}\right)$. This feature shows a good morphological correspondence with the faint IR arclike filament seen at the western section of the nebula at $\mathrm{RA}=12^{\mathrm{h}} 35^{\mathrm{m}} 10^{\mathrm{s}}$. This correspondence is also well-displayed in Fig. 10. Another structure has been noted at the northeastern region of the nebula, however it represents the remains of feature 1 at slightly more positive velocities.

Since no discernible emission of the ${ }^{13} \mathrm{CO}(2-1)$ line is detected for features 1,2 , and 3 , in order to estimate their column density and mass, we use the relation between the $\mathrm{H}_{2}$ integrated column density and the $\mathrm{CO}$ integrated emission:

$N\left(\mathrm{H}_{2}\right)=X \times \int T_{\mathrm{mb}}(\mathrm{CO}) \mathrm{d} v$,
Table 3. Physical properties estimated for the CO feature 1, feature 2, and feature 3 .

\begin{tabular}{ccccc}
\hline \hline Feature & $\begin{array}{c}\Omega \\
\left(10^{-6} \mathrm{sr}\right)\end{array}$ & $\begin{array}{c}\int T_{\mathrm{mb}}(\mathrm{CO}) \mathrm{d} v \\
\left(\mathrm{~K} \mathrm{~km} \mathrm{~s}^{-1}\right)\end{array}$ & $\begin{array}{c}N\left(\mathrm{H}_{2}\right) \\
\left(10^{20} \mathrm{~cm}^{-2}\right)\end{array}$ & $\begin{array}{c}M\left(\mathrm{H}_{2}\right) \\
\left(M_{\odot}\right)\end{array}$ \\
\hline 1 & 2.4 & 14.9 & 2.4 & 52 \\
2 & 1.3 & 12.3 & 2.0 & 24 \\
3 & 2.5 & 7.9 & 1.3 & 29 \\
\hline
\end{tabular}

where $X$ is an empirical factor that has been shown to be roughly constant for the ${ }^{12} \mathrm{CO}(1-0)$ line in Galactic molecular clouds and lies in the range of $(1-3) \times 10^{20} \mathrm{~cm}^{-2}\left(\mathrm{~K} \mathrm{~km} \mathrm{~s}^{-1}\right)^{-1}$, as estimated by the virial theorem and $\gamma$-ray emission (Bloemen et al. 1986; Solomon et al. 1987; Digel et al. 1996; Strong \& Mattox 1996). In this paper, we adopt $X=1.6 \times 10^{20} \mathrm{~cm}^{-2}\left(\mathrm{~K} \mathrm{~km} \mathrm{~s}^{-1}\right)^{-1}$ (Hunter et al. 1997). The integrated emission $\int T_{\mathrm{mb}}(\mathrm{CO}) \mathrm{d} v$ was calculated adopting an area determined by the first contour level indicated in Fig. 6. For the column density, we estimated an uncertainty of about $50 \%$, arising mostly from the factor $X$ and calibration uncertainties. The mass of the features were then estimated using Eq. (9). The obtained parameters are shown in Table 3. It is worthwhile to point out that the area and mass obtained for features 1 and 2 are likely lower limits since these features seem to be part of a larger structure (extending beyond the area covered in our APEX observations), which probably corresponds to the parental molecular cloud in which the IR bubble was formed.

\subsection{Submillimeter dust emission}

In the upper panel of Fig. 7, we present a three-color composite image of S169 as seen by Herschel images. The 70 and $160 \mu \mathrm{m}$ emissions (red and green color scales, respectively) underline the emission of the warmer dust close to the ionized gas. As can be seen from the figure, the emission at $70 \mu \mathrm{m}$ has a good morphological correspondence with the IRAC $8 \mu \mathrm{m}$ emission (black contours), although the latter exhibits a sharper edge towards the center of the nebula (see also Fig. 1), which is likely due to the destruction of the PAH molecules by the ionization front. The $70 \mu \mathrm{m}$ emission, in contrast, is more diffuse (partly because of the lower angular resolution) and extends towards the center of the nebula, which indicates that it is also tracing the warm dust that still remains mixed with the ionized gas. The same feature can be observed in the IRAC-GLIMPSE $24 \mu \mathrm{m}$ emission (not shown here). The $350 \mu \mathrm{m}$ emission (blue color scale) seems to be underlining the emission from the dust in the outer parts of the nebula. It is very likely that the emission distribution at this wavelength is mostly tracing the distribution of the cold dust immersed in the parental molecular cloud placed at the eastern border of the nebula, which is partially disclosed by features 1 and 2 (see Fig. 6).

To study the distribution of the dust temperature in the nebula, we constructed a temperature map from the ratio of the observed fluxes in two Herschel bands. Since a high level of radiative feedback from powering massive stars is usually observed in HII regions/IR bubbles, it is reasonable to assume that the molecular gas and dust are not too cold. Then we used the ratio of the 70 versus $160 \mu \mathrm{m}$ maps, which are better suited to measure color temperatures up to $\sim 80 \mathrm{~K}$. Furthermore, the angular resolution of the temperature map obtained using these bands is the highest resolution achievable from the Herschel maps. 

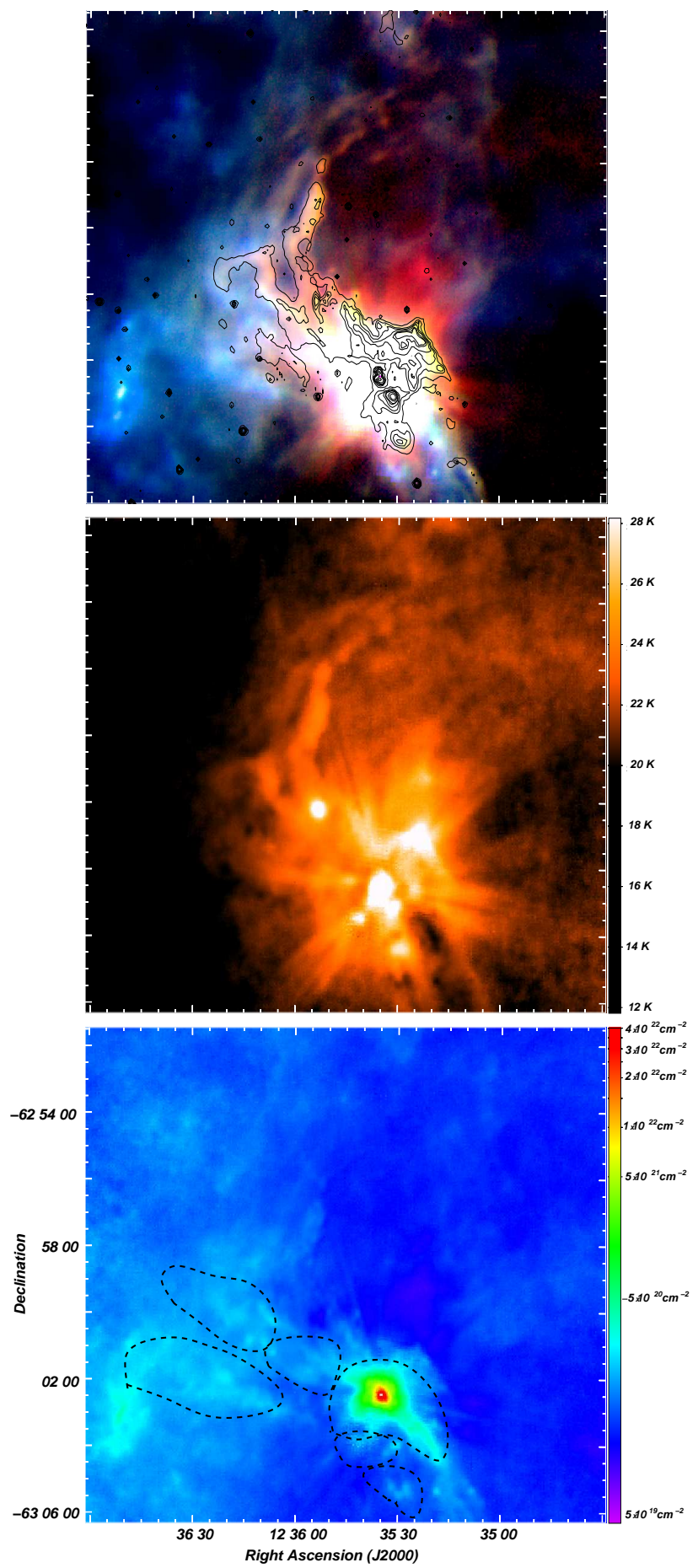

Fig. 7. Upper panel: three-color composite image of S169: Herschel PACS 70 and $160 \mu \mathrm{m}$ emission are in red and green, respectively. Herschel SPIRE $350 \mu \mathrm{m}$ emission is in blue. Black contours underline de IRAC-GLIMPSE $8 \mu \mathrm{m}$ emission at $35,55,75,105,150,170,300$, and $500 \mathrm{MJy} \mathrm{s}^{-1}$. Middle panel: dust temperature map derived from the 70 and $160 \mu \mathrm{m}$ emission. The color temperature scale is on the right. Lower panel: column density map obtained from the $160 \mu \mathrm{m}$ emission. Dashed black contours indicate the location of molecular concentrations $\mathrm{MC1}$ to MC6 for orientation

The $70 \mu \mathrm{m}$ map was smoothed down to the angular resolution of the $160 \mu \mathrm{m}$ data. Then the temperature map was constructed as the inverse function of the ratio $70-160 \mu \mathrm{m}$ maps that is, $T_{\mathrm{c}}=f_{(T)}^{-1}$. Assuming a dust emissivity following a power law of

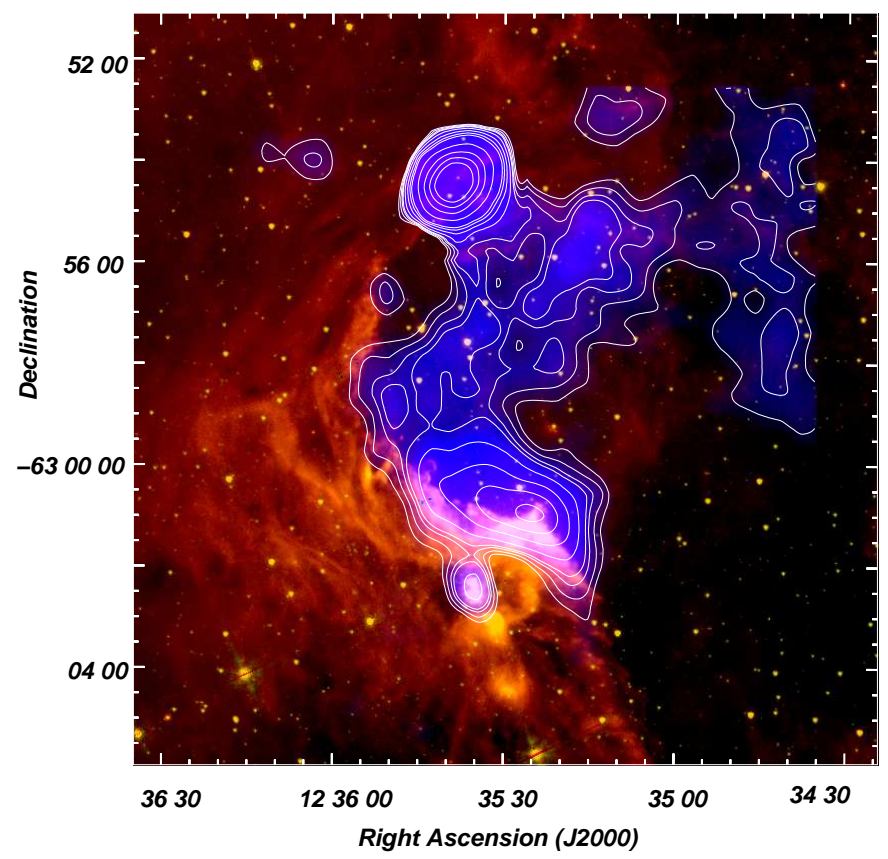

Fig. 8. Radio continuum emission distribution at $843 \mathrm{MHz}$ (blue color and white contours), superimposed on the 8 and $5.8 \mu \mathrm{m}$ emission (red and green color). Contours levels are $3.1(\sim 3 \mathrm{rms}), 4.5,6.3,7.6,12,20$, $30,40,100,200,300$, and $400 \mathrm{mJy}^{\text {beam }}{ }^{-1}$.

$\kappa_{v} \propto v^{\beta}$, with $\beta$ as the spectral index of the thermal dust emission (assumed to be $\beta=2$ ), in the optically thin thermal dust emission regime, $f_{(T)}$ takes the parametric form:

$f_{(T)}=\frac{S_{70}}{S_{160}}=\frac{B(70, T)}{B(160, T)}\left(\frac{70}{160}\right)^{\beta}$,

where $B(70, T)$ and $B(160, T)$ make up the blackbody Planck function for a temperature $T$ at wavelengths 70 and $160 \mu \mathrm{m}$, respectively. The assumption that the emission is optically thin is further justified from the derived column densities in the nebula (see Preibisch et al. 2012 for details). From the obtained map (middle panel of Fig. 7), dust temperatures between 20 and $30 \mathrm{~K}$ can be discerned. These temperatures are commonly observed in the closest regions of IR bubble nebulae (e.g., Anderson et al. 2012; Duronea et al. 2015; Figueira et al. 2017). Warmer dust is seen in the region of the denser molecular condensations (MC1, MC2, MC3, and MC4) and the border of the PDR, particularly on two spots at RA, Dec $(\mathrm{J} 2000)=$ $\left(12^{\mathrm{h}} 35^{\mathrm{m}} 24^{\mathrm{s}},-63^{\circ} 00^{\prime} 56^{\prime \prime}\right)$ and RA, Dec $(\mathrm{J} 2000)=\left(12^{\mathrm{h}} 35^{\mathrm{m}} 35^{\mathrm{s}}\right.$, $\left.-63^{\circ} 02^{\prime} 29^{\prime \prime}\right)$ where the temperature achieves $\sim 30 \mathrm{~K}$. The first spot is coincident with a bright rim placed over the southern region of the PDR and a peak in the radio continuum emission (see Fig. 8 in Sect. 4), which suggests external heating of the dust. The second spot is coincident with the molecular condensation MC3 (IRAS12326-6245) which is very likely to indicate that the dust is also internally heated. Not surprisingly, lower temperatures (12-18 K) are seen in the region of feature 3 and behind the PDR, coincident with the position of features 1 and 2.

With the temperature map, we converted the surface brightness map at $160 \mu \mathrm{m}$ into a beam averaged column density map using (Hildebrand 1983):

$N\left(\mathrm{H}_{2}\right)=R \frac{S_{160}}{\Omega_{\text {beam }} \kappa_{160} \mu m_{\mathrm{H}} B\left(160, T_{\text {dust }}\right)}$, 
where $\Omega_{\text {beam }}$ is the beam solid angle ( $\left.\pi \theta_{\mathrm{HPBW}}^{2} / 4 \ln 2\right), R$ is the gas-to-dust ratio (assumed to be 100), and $\kappa_{160}$ is the dust opacity per unit mass at $160 \mu \mathrm{m}$ assumed to be $40.5 \mathrm{~cm}^{2} \mathrm{~g}^{-1}$ (Ossenkopf $\&$ Henning 1994). The resulting map (presented in the lower panel of Fig. 7) gives an overall view of the density distribution in the whole region of the nebula. Clearly, denser regions are observed behind the PDR in the region of component A, especially towards IRAS12326-6245, where the column density achieves peak values up to $\sim 4 \times 10^{22} \mathrm{~cm}^{-2}$.

As shown in Figs. 1 and 4, the source IRAS 12326-6245 is very bright in the continuum emission at $870 \mu \mathrm{m}$ (no emission at this wavelength is detected above the $3 \mathrm{rms}$ limit outside the IRAS source). The emission at $870 \mu \mathrm{m}$ is usually optically thin and dominated by the thermal emission from dust contained in dense material (e.g., dense molecular cores or filaments). Then we used the image of ATLASGAL to more confidently derive the beam averaged density in IRAS 12326-6245 using Eq. (12). For the calculations, we adopted $\kappa_{870}=1.85 \mathrm{~cm}^{2} \mathrm{~g}^{-1}$, a value that is representative for relatively dense molecular clouds (Ossenkopf \& Henning 1994; Henning et al. 1995) and $T_{\text {dust }}=30 \mathrm{~K}$ and we obtained a column density of $N\left(\mathrm{H}_{2}\right)=8.2 \times$ $10^{23} \mathrm{~cm}^{-2}$. This value is almost two orders of magnitude higher than that observed in the column density map obtained with the emission at $160 \mu \mathrm{m}$, which might indicate that the emission is optically thick at that wavelength. Furthermore, the temperature used to derive $N\left(\mathrm{H}_{2}\right)$ in IRAS 12326-6245 $(30 \mathrm{~K})$ might have been underestimated since, as mentioned above, its emission at $160 \mu \mathrm{m}$ might not be optically thin at such high density. This could led to an overestimation of the column density in the IRAS source. The column density obtained from the 870 $\mu \mathrm{m}$ emission is also somewhat higher than those derived for $\mathrm{MC} 3$ in Sect. 3.2 from the ${ }^{13} \mathrm{CO}(2-1)$ and $\mathrm{C}^{18} \mathrm{O}(2-1)$ emissions. We do keep in mind, however, that the column density values derived from continuum IR and carbon monoxide emissions are strongly dependent on the adopted abundances (dust and carbon monoxide-to $\mathrm{H}_{2}$ ).

\section{4. lonized gas}

In Fig. 8, we show the radio continuum emission map at $843 \mathrm{MHz}$ overlaid on the 8.0 and $5.8 \mu \mathrm{m}$ emissions. Three bright structures stand out in the direction of the nebula. The brightest one $\left(S_{843}=985 \mathrm{mJy}\right)$, which does not have a discernible counterpart in the $\mathrm{H} \alpha$ emission, is observed towards the norther border of the nebula at RA, Dec $(\mathrm{J} 2000)=\left(12^{\mathrm{h}} 35^{\mathrm{m}} 39^{\mathrm{s}},-62^{\circ} 54^{\prime} 26^{\prime \prime}\right)$. Using a radio continuum image from the SGPS (not shown here), we found that this compact source has an integrated flux density of $\mathrm{S}_{1384}=0.63 \mathrm{mJy}$. Since the spectral index turns out to be $\alpha=-0.9$, we conclude that this source is extragalactic and will not be taken into further consideration.

An arc-shaped structure can be discerned towards the center of the nebula, which may be described by two main components: (i) a southern bright component with its outer edge closely delineating the PDR depicted by the $8.0 \mu \mathrm{m}$ emission; and (ii) a weaker tail about $5^{\prime}$-long extending from the northern end of the previous component towards the northwest. A number of artifacts in the radio continuum emission are obviously produced by sidelobes and grating rings from the bright extragalactic source, such as a void at RA, Dec $(\mathrm{J} 2000) \approx\left(12^{\mathrm{h}} 35^{\mathrm{m}} 45^{\mathrm{s}},-62^{\circ} 56^{\prime} 33^{\prime \prime}\right)$ and two radial strips connecting the compact source and the weak tail. Hence, we believe that the morphology of the arcshaped structure is probably distorted in the region closer to the extragalactic source. However, the morphology of the unaffected part (southernmost component) suggests that the ionized gas traced by the radio continuum emission is likely to be expanding against component $\mathrm{A}$ and features 1 and 2, supporting the same conclusion derived in Sect. 1 based on the $\mathrm{H} \alpha$ emission.

Using the integrated radio continuum flux obtained for the whole arc-shaped structure (i.e., the southern bright component and weaker tail), $S_{843}=308 \mathrm{mJy}$, we obtained its electron density and ionized mass using (Panagia \& Walmsley 1978):

$n_{\mathrm{e}}=3.113 \times 10^{2} S_{843}^{0.5} T_{\mathrm{e}}^{0.25} d^{-0.5} b\left(v, T_{\mathrm{e}}\right)^{-0.5} \theta_{\mathrm{R}}^{-1.5} \mathrm{~cm}^{-3}$

and

$M_{\text {ion }}=0.7934 S_{843}^{0.5} T_{\mathrm{e}}^{0.25} d^{2.5} b\left(v, T_{\mathrm{e}}\right)^{-0.5} \theta_{\mathrm{R}}^{1.5}(1+Y)^{-1} M_{\odot}$,

where $T_{\mathrm{e}}$ is the electron temperature (assumed to be $1 \times 10^{4} \mathrm{~K}$ ) in units of $10^{4} \mathrm{~K}, \theta_{\mathrm{R}}$ is the angular radius of the source in arc minutes (assumed to be $\left.4^{\prime}\right), b\left(v, T_{\mathrm{e}}\right)=1+0.3195 \times \log \left(T_{\mathrm{e}} /\right.$ $\left.10^{4} \mathrm{~K}\right)-0.213 \times \log (v / 1 \mathrm{GHz}), d$ is in $\mathrm{kpc}$, and $Y$ is the abundance ratio by number of $\mathrm{He}^{+}$to $\mathrm{H}^{+}$. Then we obtained $n_{\mathrm{e}}=15 \mathrm{~cm}^{-3}$ and $M_{\text {ion }}=17 M_{\odot}$. It is worthwhile pointing out that the presence of the extragalactic source projected on S169 prevents the estimation of the whole radio continuum emission related to the structure.

The number of ionizing Lyman continuum photons needed to sustain the current level of ionization in the arc-shaped structure can be calculated using (Chaisson 1976):

$N_{\text {Lyc }}=0.76 \times 10^{47} T_{\mathrm{e}}^{-0.45} S_{843} v^{0.1} d^{2} \mathrm{~s}^{-1}$,

where $T_{\mathrm{e}}$ is in units of $10^{4} \mathrm{~K}, S_{843}$ is in Jy, $v$ is in $\mathrm{GHz}$, and $d$ is in $\mathrm{kpc}$. Then, in considering distance uncertainties, we obtained for this source a range of $N_{\mathrm{Lyc}}=0.5-2.0 \times 10^{47} \mathrm{~s}^{-1}$. We keep in mind that these values are likely lower limits since about $25-50 \%$ of the UV photons are absorbed by interstellar dust in the HII region (Inoue 2001). Furthermore, Watson et al. (2008), on the basis of studies of several bubbles, concluded that $N_{\text {Lyc }}$ estimated with this method is lower than expected by about a factor of 2 . Then for the arc-shaped structure, we estimate a range of $N_{\mathrm{Lyc}} \approx(1-$ 4) $\times 10^{47} \mathrm{~s}^{-1}$. Adopting ionizing photon rates extracted from Martins et al. (2005), we estimate the spectral type of the ionizing star of S169 to be at most O9.5V. Alternatively, a handful of later B-type stars could also be responsible for powering the HII region. The third structure detected in the $843 \mathrm{MHz}$ emission is a small bright spot discernible in the southern region of the nebula at RA, Dec $(\mathrm{J} 2000)=\left(12^{\mathrm{h}} 35^{\mathrm{m}} 35^{\mathrm{s}},-63^{\circ} 02^{\prime} 29^{\prime \prime}\right)$, which is certainly the radio continuum counterpart of IRAS 12326-6245. Using the flux density obtained for this source $\left(S_{843}=12 \mathrm{mJy}\right)$, we obtained $n_{\mathrm{e}}=70 \mathrm{~cm}^{-3}, M_{\text {ion }}=0.3 M_{\odot}$, and $N_{\text {Lyc }}=(0.4-$ 1.4) $\times 10^{46} \mathrm{~s}^{-1}$. It is worthwhile pointing out that for the previous estimations, we assumed optically thin emission at $843 \mathrm{MHz}$; since that assumption cannot be verified, estimations obtained with the Eqs. (13)-(15) should be taken as the lower limits. In fact, for the case of the radio continuum emission associated with IRAS 12326-6245, Dedes et al. (2011) used cm observations obtained by Urquhart et al. (2007) to derive a Lyman continuum flux of $8.2 \times 10^{48} \mathrm{~s}^{-1}$, which is almost three orders of magnitude larger than the value obtained with Eq. (15) for the source.

In a broad sense, the distribution of the ionized gas inside the cavity suggests an electron density gradient, with the densest material near the border of the IR nebula. This configuration suggests that the nebula is bounded by density to the west, and bounded by ionization to the east, north, and south. Furthermore, the structure detected to the west of the nebula along 


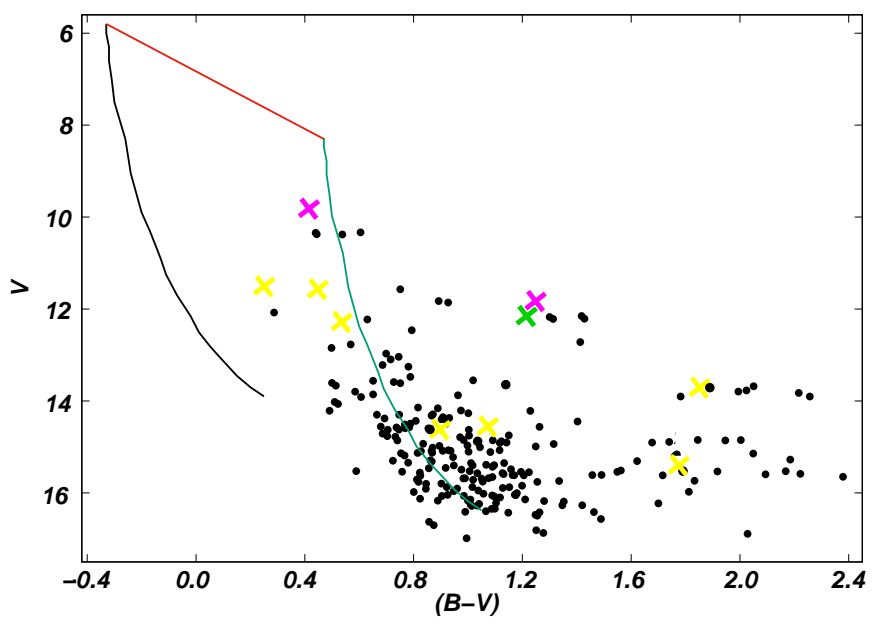

Fig. 9. Color magnitude diagram of stars searched in a region centered in S169. Black dots are young spectral type stars with different reddening values (see text in Sect. 5). Magenta, green, and yellow crosses represent the $\mathrm{O} 9 \mathrm{~V}, \mathrm{~B} 1 \mathrm{~V}$, and $\mathrm{B} 2 \mathrm{~V}$ (or later) stars, all of them ionizing candidate stars displayed with the same colors in Fig. 10. Green and black curves represent the MS shifted according to the distance modulus adopted with and without reddening, respectively. Red line indicates the normal reddening path $\left(R_{\mathrm{v}}=3.1\right)$.

RA $\sim 12^{\mathrm{h}} 34^{\mathrm{m}} 44^{\mathrm{s}}$, which appears connected with the eastern arcshaped structure within the $3 \mathrm{rms}$ emission limit, might be indicative of a "champagne-flow" scenario (Tenorio-Tagle 1979).

\section{Stellar and protostellar content in the nebula}

\section{1. lonizing star candidates}

With the aim of identifying possible ionizing stars in the nebula, we first inspected IR catalogs in a region of $\sim 7^{\prime}$ in radius centered on RA, Dec $(J 2000) \approx\left(12^{\mathrm{h}} 35^{\mathrm{m}} 28^{\mathrm{s}},-62^{\circ} 58^{\prime} 04^{\prime \prime}\right)$. Making use of GLIMPSE data, we identified Class III candidates, which are usually referred to as main-sequence (MS) young stars using color-color diagrams (Allen et al. 2004). Then we cross-correlated these sources with sources selected from the 2MASS catalog with photometric uncertainty, $K_{s}<$ 0.05 mag (Ducati et al. 2001), and we obtained $\sim 600$ Class III sources with reliable $J H K$ photometry. Since none of these sources were found to have spectral classification, we used the parameter $Q_{\mathrm{NIR}}=(J-H)-1.7\left(H-K_{s}\right)$ following Borissova et al. (2011) to separate sources between early (O-B) MS stars $\left(-0.1<Q_{\mathrm{NIR}}<0.15\right)$ and stars that reveal IR excess $\left(Q_{\mathrm{NIR}}<-0.1\right)$ and are likely preMS stars or YSOs, or both. Then we obtained 60 MS candidates.

Next, we inspected visual catalogs in the same region. A search in the APASS and ASCC-2.5 V3 catalogs yielded 228 and 10 sources, respectively, both with $\mathrm{V}$ and $\mathrm{B}$ magnitudes. We also carried out a search in the astrometric catalog Gaia DR2 of more than 6000 sources with parallax data in the region of S169. Thereafter, we cross-correlated all sources found with the APASS, Gaia DR2, and ASCC-2.5 catalogs, obtaining only 90 sources in common between the first two catalogs, which includes the 10 sources of the ASCC- 2.5 catalog. We then estimated the distance of visual sources employing the colormagnitude ( $B-V$ vs. $V$ ) diagram (see Fig. 9), calibrated with the optical $(B V)$ (Schmidt-Kaler Th. 1982) to adjust the theoretical MS by a distance of $\sim 2 \mathrm{kpc}$ (see Sect. 6.2) and color excess $\mathrm{E}(B-V)=0.8$ magnitudes. Thus, we were able to identify the stars with photometric distance close to the nebula. Among these sources, only 19 had distances between 1.7 and $2.3 \mathrm{kpc}$ that were estimated from the parallax catalog from Gaia.

In the next step, we cross-correlated the $60 \mathrm{MS}$ candidates obtained from the IR data with the sources previously studied with a color magnitude diagram and we found 47 sources in common. In order to better determine their spectral types, we inspected their loci in a $(J-H)$ vs. $(H-K)$ diagram adopting the MS calibration given by Schmidt-Kaler (Th. 1982), Cousins (1978), and Koornneef (1983), the absorption ratios $\left(r_{x}=A_{x} / A_{v}\right)$ given by Rieke \& Lebofsky (1985), van der Hulst curve 15, and the color transformations from 2MASS to Koorneef data of Carpenter et al. (2001).

Finally, we kept only those stars that are projected onto the cavity of the bubble $\left(\sim 3^{\prime}\right.$ in radius centered on RA, Dec $\left.(\mathrm{J} 2000) \approx\left(12^{\mathrm{h}} 35^{\mathrm{m}} 28^{\mathrm{s}},-62^{\circ} 58^{\prime} 04^{\prime \prime}\right)\right)$. This yielded only 10 sources with spectral types O9 (2 sources), B1 (1 source) and B2 or later (7 sources), all of them probable MS stars; it is worthwhile pointing out that only 5 of these sources belong to the group of 19 sources with distances between 1.7 and $2.3 \mathrm{kpc}$ obtained from Gaia. The positions of these sources in the MC diagram and in the cavity are shown in Figs. 9 and 10, respectively.

\subsection{Identification of candidate YSOs}

Making use of the MSX, WISE and Spitzer point source catalogs, we look for the primary tracers of stellar formation activity onto the molecular clouds related to S169. To this aim, MSX sources were selected satisfying a flux quality $Q>1$ in the four bands. WISE sources with photometric flux uncertainties $>0.2 \mathrm{mag}$ and signal-to-noise ratio $(\mathrm{S} / \mathrm{N})<7$ in the W1, W2, W3, and W4 bands, were rejected. Finally, we kept Spitzer sources with photometric uncertainties $<0.2$ mag in the four IRAC bands. Then, within a $14^{\prime}$ box centered at RA, Dec $(J 2000)=\left(12^{\mathrm{h}} 35^{\mathrm{m}} 44^{\mathrm{s}},-63^{\circ} 00^{\prime} 08^{\prime \prime}\right)$, we found a total of 2 MSX, 400 WISE, and 810 Spitzer sources fulfilling the selection criteria mentioned above. To identify candidate YSOs we adopted the classification scheme described in Lumsden et al. (2002), Koenig et al. (2012), and Gutermuth et al. (2009) for the MSX, WISE, and Spitzer sources, respectively. Several sources were found to qualify the above criteria, which are listed in Table A.1. The table presents the designation of the sources, their coordinates, flux densities, information concerning the type of the protostellar object, matching with another source in the table, and coincidence with a molecular or IR structure.

Among the MSX sources, we found one MYSO candidate, which coincides with the center of IRAS 12326-6245, and one CHII region candidate, which coincides with a WISE Class II candidate (see below). Before attempting to identify the candidate YSOs from the listed WISE and Spitzer sources, we selected the non-YSO sources with excess infrared emission, such as PAH emitting galaxies, broad line active galactic nuclei (AGNs), unresolved knots of shock emission, and PAH emission features. Then, a total of 89 and 51 WISE and Spitzer sources, respectively, were dropped from the lists. Among the remaining 311 WISE and 759 Spitzer sources, 12 (5 WISE and 7 Spitzer) were identified as Class I sources (i.e., sources with IR emission arising mainly from a dense infalling envelope, including flat spectrum objects) and 60 (43 WISE and 17 Spitzer) as Class II sources (i.e., preMS stars with optically thick disks).

The location of the identified candidate YSOs is shown in Fig. 10, where the 8.0 and $5.8 \mu \mathrm{m}$ emissions together with the 


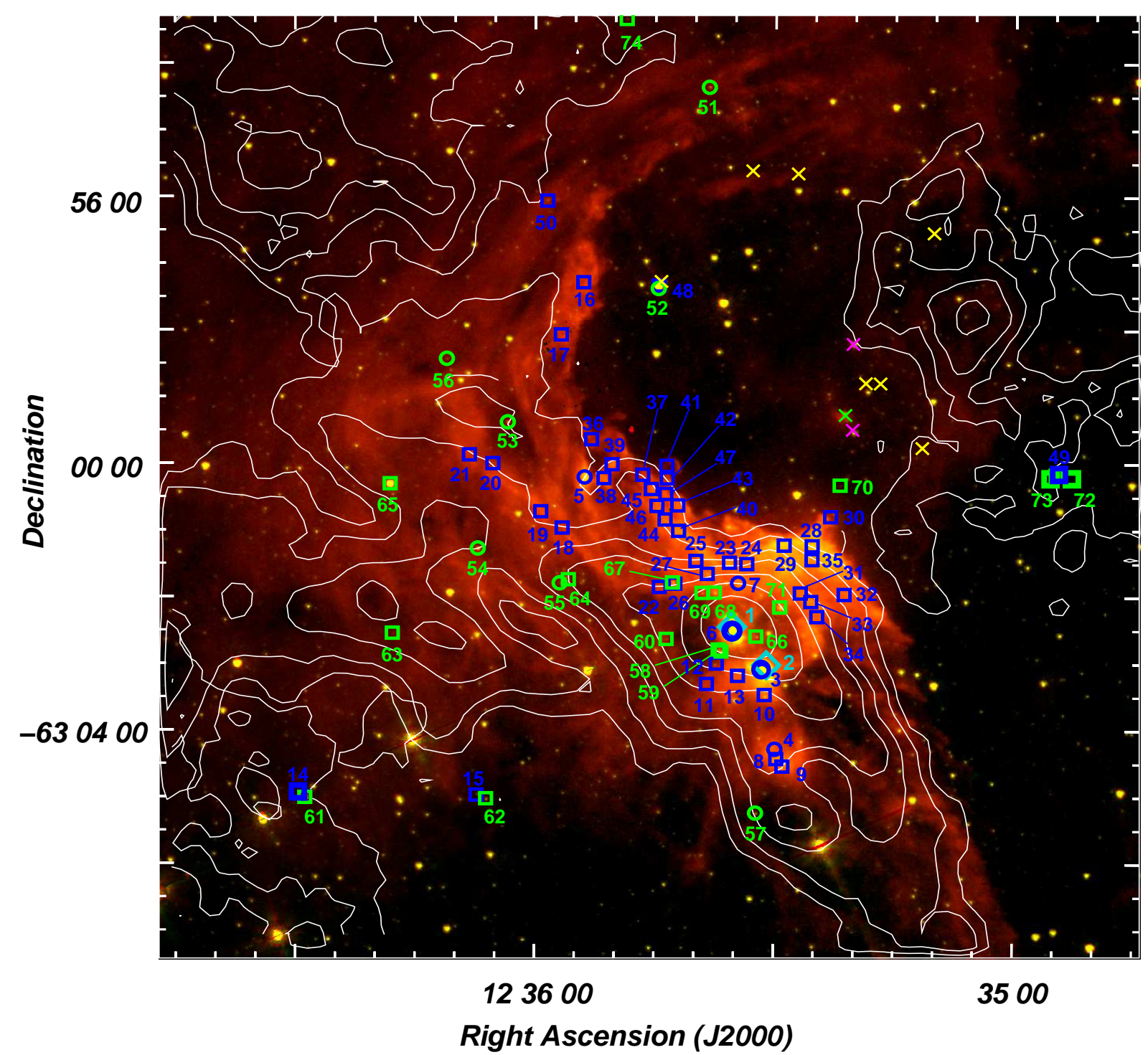

Fig. 10. MSX, WISE, and Spitzer candidate YSOs projected onto the IRAC-GLIMPSE image at 8.0 and $5.8 \mu \mathrm{m}$. Cyan diamonds indicate the MSX sources, blue circles and blue squares indicate WISE Class I and Class II sources, respectively, and green circles and green squares indicate the Spitzer Class I and Class II sources, respectively. The numerical references of YSOs are based on those from Table A.1. The size of the symbols do not necessary match the angular size of the sources. Ionizing candidate stars identified in Sect 5.1 are also indicated by crosses in magenta $(\mathrm{O} 9 \mathrm{~V})$, green $(\mathrm{B} 1 \mathrm{~V})$, and yellow $\left(\mathrm{B} 2 \mathrm{~V}\right.$ or later). White contours indicate the ${ }^{13} \mathrm{CO}$ emission of component $\mathrm{A}$ (first contour level $\left.\sim 8 \mathrm{rms}\right)$ and the $\mathrm{CO}$ emission of feature 1 (first contour level $\sim 13 \mathrm{rms}$ ), feature 2 (first contour level $\sim 12 \mathrm{rms}$ ), and feature 3 (first contour level $\sim 10 \mathrm{rms}$ ).

emission of both the ${ }^{13} \mathrm{CO}$ and $\mathrm{CO}$ structures (i.e, component A and features 1, 2, and 3) are also displayed. As expected, the bulk of the candidate YSOs are seen projected onto the densest molecular gas (component A). A noticeable feature is the concentration of sources along the two brightest PDR areas which, as mentioned in Sect. 3.2, delineates the borders of the molecular condensations. Namely, the WISE sources \#5, \#23, \#24, \#25, $\# 27, \# 28, \# 29, \# 30, \# 32$, and \#35 to \#47, seem to be related to the PDR closer to the ionized gas bordering the molecular emission of MC3 and MC4 (noted as (+) in the last column of Table A.1), while WISE sources \#7, \#18, \#19, \#20, \#21, \#22, and \#26, and Spitzer sources \#54, \#65, \#67, \#68, and \#69 lie along the PDR that lies farther away from the ionized gas and related to the densest parts of MC3, MC4, and MC5 (noted as (*) in Table A.1). Not surprisingly, another appreciable concentration of candidate YSOs, namely WISE sources \#3, \#6, \#10, \#11, \#12, and \#13, Spitzer sources \#58, \#59, \#60, \#66, and \#71, and MSX sources \#1 and \#2 can be noticed in the region of the molecular concentration MC3.

\section{Discussion}

\subsection{A model for S169 and its molecular environment}

Since IR bubbles are believed to be born within dense molecular clouds, the classical scenario predicts that, in a uniform medium, the molecular gas around the bubble should expand spherically. Under that assumption, a molecular shell with a central velocity $V_{0}$ and an expansion velocity $V_{\text {exp }}$ should depict in a position-position diagram a "disk-ring" pattern when observed at different velocities. At $V_{0}$ (corresponding to the systemic velocity of the bubble), the shell should attain its maximum diameter; whereas, at extreme velocities (either negative or positive with respect to $V_{0}$ ), the molecular emission should resemble a disk. 


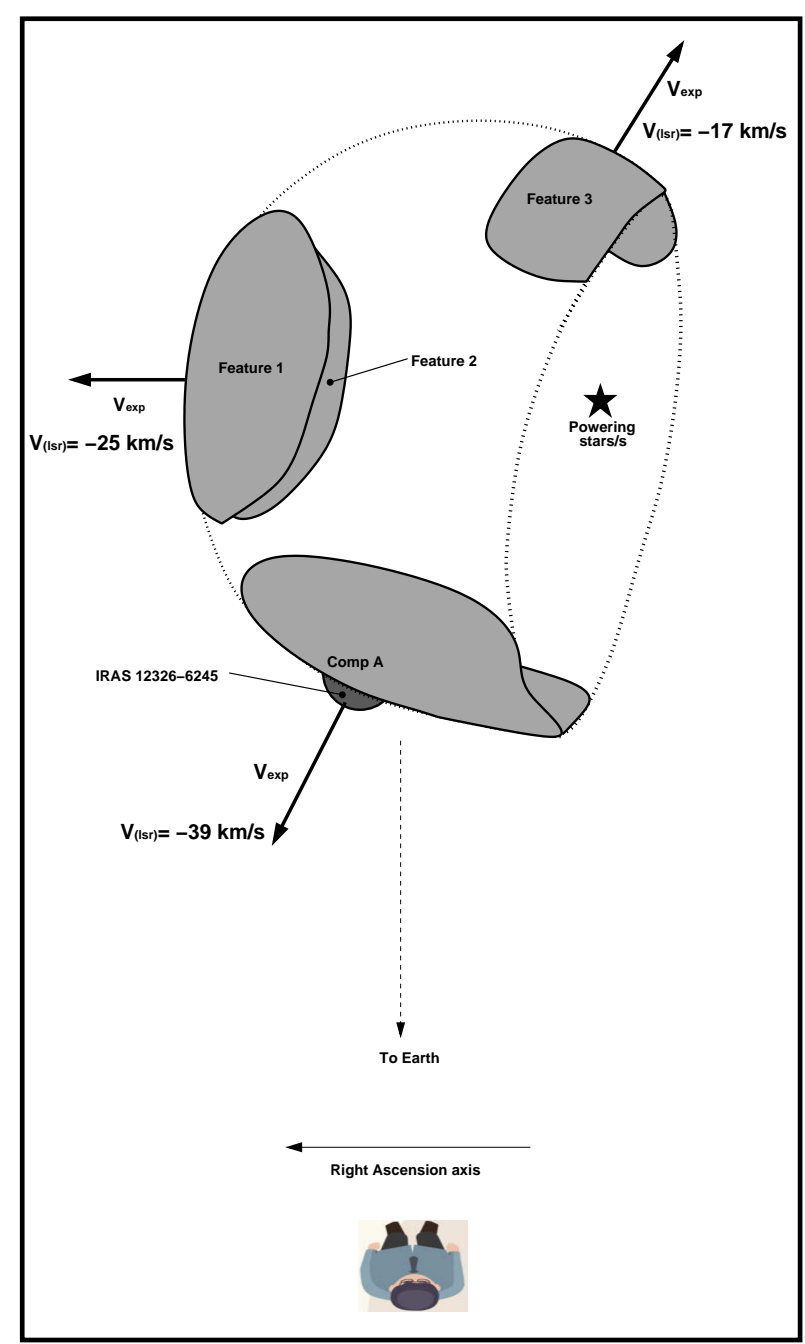

Fig. 11. Simple sketch of the proposed model to explain the morphology and velocity of the molecular gas associated with S169. The right ascension axis, as visualized by the observer, is indicated as reference (the declination axis is perpendicular to the plane of the sketch, increasing towards the reader).

At intermediate velocities, the radius of the ring shrinks as the extreme velocity is approached. Although these features were never observed all together in the molecular gas around IR bubbles, the behavior of the molecular gas around S169 certainly clashes with that of the classical IR bubble model.

In order to address the observed characteristics of the molecular gas associated with S169, we present a simple model taking into account its morphology, relative position with respect to the IR and $\mathrm{H} \alpha$ emissions and the velocity intervals as constraints. Needless to say, we assume that component A, feature 1 , feature 2, and feature 3 are physically associated with S169, a well-grounded conclusion given their good morphological correspondence with the IR nebula. Hence, we propose that component $\mathrm{A}$ and features 1, 2, and 3 could be explained in terms of an expanding, partially complete semispherical structure. A simple sketch is presented in Fig. 11, where the right ascension axis is indicated for the sake of clarity (the declination axis is then perpendicular to the sketch, increasing towards the reader). The expansion of the molecular gas is then revealed by the velocity of the different molecular structures. The molecular gas approaching in the direction of Earth is depicted by the molecular structure at more negative velocity (component A) with an approaching velocity of $V_{\text {appr }} \approx-39 \mathrm{~km} \mathrm{~s}^{-1}$, while the molecular gas receding is depicted by the structure at more positive velocity (feature 3 ) with a receding velocity of $V_{\text {rec }} \approx-17 \mathrm{~km} \mathrm{~s}^{-1}$. Molecular structures at intermediate velocities (features 1 and 2) thus represent the molecular gas at $V_{0} \approx-25 \mathrm{~km} \mathrm{~s}^{-1}$ expanding approximately in perpendicular directions with respect to the direction of Earth. A glance at Fig. 6 suggests that the expansion velocity of features 1 and 2 might have components in the direction of the declination axis, which is positive for the case of feature 1 and negative for the case of feature 2 (this cannot be depicted in the two-dimension sketch presented in Fig. 11).

Therefore, with this simple model we are able to explain not only the morphology and kinematics of the molecular gas, but also the characteristics of the ionized gas (traced by the radio continuum emission), which seems to be expanding against the molecular gas towards the east, north, and south, while it is expanding freely to the west, probably in a sort of a champagne-flow effect.

According to the model, a rough estimation of the expansion velocity of the molecular gas around S169 may be simply obtained by:

$V_{\text {exp }}=\frac{\left|V_{\text {appr }}\right|-\left|V_{\text {rec }}\right|}{2 \times \cos (\theta)}$,

with $\theta$ as the angle between the line of sight and the direction of $V_{\text {exp }}$ of component A and feature 2. Based on the morphology of the IR nebula, a conservative value $\theta=25^{\circ}$ can be adopted, and the obtained expansion velocity turns out to be $V_{\exp } \sim 12 \mathrm{~km} \mathrm{~s}^{-1}$. Having the expansion velocity, the dynamical age of the nebula, $t_{\text {dyn }}$, can be derived considering a radius of $2.3 \pm 0.8 \mathrm{pc}\left(\sim 4^{\prime}\right.$ at a distance of $\left.2.03_{-0.61}^{+0.77} \mathrm{kpc}\right)$, which turns out to be in the range of $(1.2-2.6) \times 10^{5} \mathrm{yr}$.

\subsection{Distance to S169 and IRAS 12326-6245}

As noted in Sect. 1, the adopted distance in the literature for IRAS $12326-6245$ is $4.4 \mathrm{kpc}$. This value was first estimated by Osterloh et al. (1997), who performed a study of a number of southern IRAS sources using IR continuum and millimeter line data. The authors adopted distances that were taken from the literature where possible (either directly or by kinematic and spatial association with another source of known distance). Otherwise, kinematical distances were determined using the central velocity of the $\mathrm{CS}(2-1)$ or $\mathrm{CO}(2-1)$ emission lines and the Galactic rotation curve of Clemens (1985). For the case of IRAS 12326-6245, a kinematical distance could not be determined by the authors since the central velocity of the CS and CO lines $\left(-39.4 \mathrm{~km} \mathrm{~s}^{-1}\right.$ and $-39.7 \mathrm{~km} \mathrm{~s}^{-1}$, respectively) are forbidden according to the Galactic rotation model. Then the authors adopted for IRAS 12326-6245 the distance to the source IRAS $1283-6128(4.4 \mathrm{kpc})$, which is kinematically close to within $0.5 \mathrm{~km} \mathrm{~s}^{-1}$ as well as spatially to within $2^{\circ}$.

In Sect. 3.1, we offer a confirmation of the physical association between S169 and IRAS 12326-6245. Furthermore, according to the model proposed in Sect. 6.1, the source IRAS 123266245 (embedded in component A) is expanding at a velocity coarsely estimated as $V_{\exp } \sim 12 \mathrm{~km} \mathrm{~s}^{-1}$ with respect to the systemic velocity of the bubble $\left(V_{0} \approx-25 \mathrm{~km} \mathrm{~s}^{-1}\right)$. Assuming that our model is well-suited to explain the characteristics of the molecular gas around the bubble nebula, this leads to the 


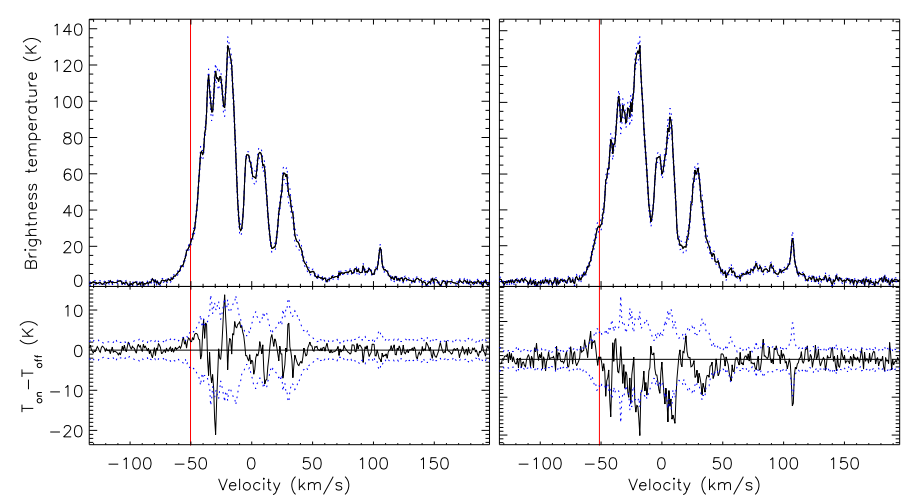

Fig. 12. Left panel: HI emission/absorption profile towards the bright southern component of the radio continuum arc-shaped feature. Right panel: same for the extragalactic source at the north of the nebula. The error envelopes are plotted with blue dotted lines. Vertical red lines indicate the velocity of the tangent point.

unavoidable conclusion that the velocity reported in the literature for IRAS 12326-6245 (approximately $-39 \mathrm{~km} \mathrm{~s}^{-1}$ ) does not represent the velocity of the ISM in the surroundings of both, the IRAS source and S169. This is understandable considering that previous studies of IRAS 12326-6245 were not performed in the whole context of the nebula S169, but rather focused only on the IRAS source. Instead, we believe that the velocity of features 1 and 2 (approximately $-25 \mathrm{~km} \mathrm{~s}^{-1}$ ), corresponding approximately to the systemic velocity of the bubble nebula, is more suitable for determining the kinematical distance for both, S169 and IRAS 12326-6245. Furthermore, in a study of the rotation curve in the Southern Galaxy, Alvarez et al. (1990) found an excess anomally of $+12.2 \mathrm{~km} \mathrm{~s}^{-1}$ in the terminal velocities in the Galactic longitude range between $l=280$ and $l=312$, with respect to the trend in the rest of the IV Galactic quadrant. This excess includes the region studied here since we have our source at Galactic coordinates $(l, b)=(301.134,-0.225)$. Next, by adopting a velocity of $-25 \mathrm{~km} \mathrm{~s}^{-1}$, and using the rotation curve of Reid et al. (2014) with the Monte Carlo method ${ }^{14}$ (see Wenger et al. 2018 for details) and a conservative velocity uncertainty of $1 \mathrm{~km} \mathrm{~s}^{-1}$, two kinematical distances are obtained, namely: a near kinematical distance of $2.03_{-0.61}^{+0.77} \mathrm{kpc}$, and a far kinematical distance of $6.59_{-0.77}^{+0.62} \mathrm{kpc}$.

To resolve the twofold ambiguity in kinematic distance, we made use of the HI data from the SGPS constructing absorption profiles towards the bright southern component of the radio continuum arc-shaped feature and, for comparison, towards the extragalactic source (see Sect. 4). The radio continuum data were convolved to a beam of $2^{\prime}$ to make them compatible with the resolution of the HI data. The expected off-source profile was computed using a bilinear interpolation method as described in Reynoso et al. (2017). The results are shown in Fig. 12. From the emission spectra, the tangent point lies at approximately $-50 \mathrm{~km} \mathrm{~s}^{-1}$ (indicated with red vertical lines in Fig. 12), while emission at the outer Galaxy is observed as far as approximately $110 \mathrm{~km} \mathrm{~s}^{-1}$. The extragalactic source (right panel) exhibits, as expected, absorption features up to approximately $-45 \mathrm{~km} \mathrm{~s}^{-1}$, coincident with the tangent point within a few $\mathrm{km} \mathrm{s}^{-1}$, a difference that can be ascribed to the ISM turbulence. Another clear absorption feature at approximately $110 \mathrm{~km} \mathrm{~s}^{-1}$ confirms the extragalactic nature of this source. In contrast, the profile corresponding to the arc-shaped feature (left panel) displays

14 https://www.treywenger.com/kd/index.php the last significant absorption feature at about approximately $-30 \mathrm{~km} \mathrm{~s}^{-1}$. Considering the absence of absorption in a gap of about $20 \mathrm{~km} \mathrm{~s}^{-1}$ between this velocity and the tangent point, it is very unlikely that S169 is located at the far side of the Solar circle. Based on these results, we conclude that the near kinematical distance $\left(2.03_{-0.61}^{+0.77} \mathrm{kpc}\right)$ is the most adequate for S169. The detection of $\mathrm{H} \alpha$ emission at the center of the nebula (see Fig. 1), which is likely the optical counterpart of the ionized nebula, gives more support to this conclusion since the absorption that could be produced by the ISM along the far kinematical distance $(\sim 6.6 \mathrm{kpc})$ would make the $\mathrm{H} \alpha$ emission much fainter or even undetectable. Furthermore, the whole molecular cloud that contains IRAS 12326-6245 has been identified to be in the near side of the Carina Spiral Arm by Cohen et al. (1985).

\subsection{Possible triggered star formation}

The spatial distribution of the candidate YSOs along the borders of the IR nebula and the densest part of the molecular gas (see Sect. 5.2) is indicative of a triggered star-forming process such as collect-and-collapse (C\&C; Elmegreen \& Lada 1977) or radiative driven implosion (RDI; Lefloch \& Lazareff 1994) might be acting on the nebula.

In order to test the $\mathrm{C} \& \mathrm{C}$ mechanism, we use the classical model of Whitworth et al. (1994) for expanding HII regions. Then, we estimated the fragmentation time, $t_{\text {frag }}$, and the radius of the HII region when the fragmentation occurs, $R_{\mathrm{frag}}$, as:

$t_{\text {frag }}=1.56 a_{0.2}{ }^{7 / 11} n_{3}^{-5 / 11}\left(N_{49}\right)^{-1 / 11} 10^{6} \mathrm{yr}$,

$R_{\text {frag }}=5.8 a_{0.2}^{4 / 11} n_{3}^{-6 / 11}\left(N_{49}\right)^{1 / 11} \mathrm{pc}$,

with $a_{0.2}$ as the isothermal sound speed in the compressed layer in units of $0.2 \mathrm{~km} \mathrm{~s}^{-1}\left(a_{\mathrm{s}} / 0.2 \mathrm{~km} \mathrm{~s}^{-1}\right), n_{3}$ the surrounding homogeneous infinite medium into which the HII region expands, in units of $10^{3} \mathrm{~cm}^{-3}\left(n_{0} / 10^{3} \mathrm{~cm}^{-3}\right)$, and $N_{49}$ the number of ionizing Lyman continuum photons, in units of $10^{49} \mathrm{~s}^{-1}\left(N_{\mathrm{Lyc}}^{*} / 10^{49} \mathrm{~s}^{-1}\right)$. We adopt $a_{\mathrm{s}}=0.2 \mathrm{~km} \mathrm{~s}^{-1}$ for the collected layer (likely a lower limit since both turbulence and extra heating from intense subLyman continuum photons leaking from the HII region could increase this value) and $N_{\text {Lyc }}^{*}=2.5 \times 10^{47} \mathrm{~s}^{-1}$ (see Sect. 4). To estimate the initial density, $n_{0}$, we averaged the total mass of the molecular gas around S169 $\left(\sim(7.4 \pm 4.4) \times 10^{3} M_{\odot}\right)$ over a sphere of $2.3 \pm 0.8 \mathrm{pc}$ in radius, which yields a density in the range of $n_{0}=(0.35-12) \times 10^{3} \mathrm{~cm}^{-3}$. Thus, we obtain $t_{\text {frag }}$ in the range between 0.5 and $2.2 \mathrm{Myr}$ and $R_{\text {frag }}$ between 1.1 and $7.4 \mathrm{pc}$.

A comparison between $R_{\text {frag }}$ and the present radius of the nebula suggests (within errors) that the $C \& C$ process may be acting in S169. On the contrary, the estimated fragmentation time is higher than the $t_{\mathrm{dyn}}$ derived for the region in Sect. 6.1. Thus, there is no conclusive evidence to support that protostellar objects at the borders of S169 could have been formed as a result of the C\&C mechanism. We ought to keep in mind, however, that the model of Whitworth et al. (1994) is valid for evolution of the HII regions only in a uniform molecular environment, which is certainly not the case with S169.

Regarding the possibility that the RDI process is taking place in certain regions of the nebula, a more detailed study is required, with better angular resolutions of both the molecular and radio continuum data. Nevertheless, we can clearly conclude that the presence of the intense PDR emission detected as a bright rim of the densest molecular feature suggests that the RDI process could be responsible for the formation of some candidate YSOs. 
In fact, an alternative scenario for the star formation in S 169 could be that proposed by Walch et al. (2015), which considers the action of massive stars on a fractal molecular cloud resulting in a hybrid mechanism that combines elements of C\&C and RDI and which has the capability to generate shell structures with dense clumps within them. If this were the case for S169, the denser region where IRAS 12326-6245 is located could be explained by considering an initial non-uniform molecular cloud with a significant increase in density and, thus, the RDI process could be the main action responsible for the formation of the candidate YSOs located behind this border and, in particular, for the IR source.

\section{Summary}

As part of a broader project aimed at characterizing and studying the physical properties of Galactic IR bubbles and their surroundings, we present a multiwavelength analysis of the southern IR dust bubble S169 associated with the massive star forming core IRAS 12326-6245. To analyze the characteristics of the molecular gas in the whole nebula, we use $\mathrm{CO}(2-1),{ }^{13} \mathrm{CO}(2-1)$, $\mathrm{C}^{18} \mathrm{O}(2-1)$ line data, while for the IRAS source we also use $\mathrm{HCN}(3-2)$, and $\mathrm{HCO}^{+}(3-2)$ data, all obtained with the APEX telescope. To study the characteristics of the dust and ionized gas, as well as to investigate the presence of stellar and protostellar objects in the nebula, we use archival data.

The analysis of the $\mathrm{CO}(2-1),{ }^{13} \mathrm{CO}(2-1)$, and $\mathrm{C}^{18} \mathrm{O}(2-1)$ data allows us to identify the molecular gas linked to the nebula. We report three molecular components at approximately -39 , -25 , and $-17 \mathrm{~km} \mathrm{~s}^{-1}$ (components $\mathrm{A}, \mathrm{B}$, and $\mathrm{C}$ ), which are morphologically correlated with different regions of the IR nebula. The component at $-39 \mathrm{~km} \mathrm{~s}^{-1}$ (component A) is associated with the brightest part of the IR nebula (at the southern region) and is the only one detected in ${ }^{13} \mathrm{CO}(2-1)$ and $\mathrm{C}^{18} \mathrm{O}(2-1)$ emissions. For this component, we have identified six molecular condensations (namely MC1, MC2, MC3, MC4, MC5, and MC6) based on the ${ }^{13} \mathrm{CO}$ and $\mathrm{C}^{18} \mathrm{O}$ emissions. These condensations have masses between $\sim 90$ and $5500 M_{\odot}$, and $\mathrm{H}_{2}$ column densities between $\sim 6 \times 10^{21}$ and $1 \times 10^{23} \mathrm{~cm}^{-2}$. The densest one (MC3) is the molecular counterpart of IRAS 12326-6245, which demonstrates the physical association between the nebula and the IRAS source. An LTE analysis of the $\mathrm{HCO}^{+}(3-2)$ and $\mathrm{HCN}(3-2)$ lines on this source, assuming 50 and $150 \mathrm{~K}$, respectively, indicates column densities of $N\left(\mathrm{HCO}^{+}\right)=(5.2 \pm 0.1) \times 10^{13} \mathrm{~cm}^{-2}$ and $N(\mathrm{HCN})=(1.9 \pm 0.5) \times 10^{14} \mathrm{~cm}^{-2}$. For this condensation, an $\mathrm{H}_{2}$ column density up to $\sim 8 \times 10^{23} \mathrm{~cm}^{-2}$ is obtained from the emission at $870 \mu \mathrm{m}$.

The molecular components at -25 and $-17 \mathrm{~km} \mathrm{~s}^{-1}$ (components $\mathrm{B}$ and $\mathrm{C}$ ) seem to be associated with the faintest and more external regions of the IR nebula. Unlike component A, components $\mathrm{B}$ and $\mathrm{C}$ are not detected in ${ }^{13} \mathrm{CO}$ and $\mathrm{C}^{18} \mathrm{O}$ emissions, which indicates that they are composed of low-density gas. This can be confirmed from Herschel images at 70, 160, and $350 \mu \mathrm{m}$.

With the purpose of explaining the spatial distribution and velocity of components $\mathrm{A}, \mathrm{B}$, and $\mathrm{C}$, we propose a very simple model, which consists of a partially complete semisphere structure expanding at $\sim 12 \mathrm{~km} \mathrm{~s}^{-1}$. According to the model, component A represents the molecular gas approaching at a velocity of $-39 \mathrm{~km} \mathrm{~s}^{-1}$, while component $\mathrm{C}$ (feature 3 ) the molecular gas receding at a velocity of $-17 \mathrm{~km} \mathrm{~s}^{-1}$. The systemic velocity of the molecular gas associated with the bubble is then $-25 \mathrm{~km} \mathrm{~s}^{-1}$, which is the velocity of component B (features 1 and 2). The distribution in the radio continuum emission at $843 \mathrm{MHz}$ suggests an HII region bounded by ionization to the east, north and south, and by density to the west. This appears to be in line with the model proposed for the molecular gas. The model proposed for the molecular gas brings an additional discussion about the distance of IRAS 12326-6245 (and consequently S169) since the radial velocity adopted in the literature for the IRAS source (approximately $-39 \mathrm{~km} \mathrm{~s}^{-1}$ ) would not be representative of the velocity of the ISM in its surroundings. Instead, we believe that the systemic velocity of the bubble $\left(-25 \mathrm{~km} \mathrm{~s}^{-1}\right)$ is more adequate for determining its kinematical distance. Then, using the Galactic rotation model and HI absorption profiles, we determine a kinematical distance of $2.03_{-0.61}^{+0.77} \mathrm{kpc}$ for S169 and IRAS 12326-6245.

Using point source catalogs, we identify 10 ionizing stars candidates projected onto the cavity. They have spectral types between $\mathrm{O} 9 \mathrm{~V}$ and $\mathrm{B} 2$, which are necessary to sustain the current level of ionization of the HII region. We also identify a number of candidate YSOs projected mostly onto component A, more precisely: MC3, MC4, and MC5, confirming that active star formation has developed along the borders of the bubble. After comparing the fragmentation time and fragmentation radius with the age and current radius of the nebula, we cannot assert that the $\mathrm{C} \& \mathrm{C}$ process is acting in the collected layers of gas at the edge of the bubble. We do keep in mind, however, the limitations of the models applied to S169 and the possibility that other triggering star-forming processes, such as RDI or a combination of both, $\mathrm{RDI}$ and $\mathrm{C} \& \mathrm{C}$, could be acting in the region.

In summary, the infrared bubble S169 is a relatively young HII region that has profoundly affected its surroundings, creating a molecular shell that continues to possess a large expanding motion and where several condensations host candidate protostellar objects in their dense interiors, with the source IRAS 12326-6245 standing out as the most striking among them.

Acknowledgements. We would like to thank the anonymous referee for his/her helpful comments and suggestions that led to the improvement of this paper. N.U.D and M.A.C acknowledge support from UNLP, PPID G005 and CONICET grant PIP 112-201701-00507 (Argentina). L.B. and R.F. acknowledges support from CONICYT project Basal AFB-170002. E.M. acknowledges support from the Brazilian agency CNPq (grant 150465/2019-0). L.A.S acknowledges support from UNLP PPID G005 grant. S.C., L.A.S. and E.M.R. are partially funded by CONICET grant PIP 112-201701-00604 (Argentina).

\section{References}

Allen, L. E., Calvet, N., D’Alessio, P., et al. 2004, ApJS, 154, 363

Alvarez, H., May, J., \& Bronfman, L. 1990, ApJ, 348, 495

Anderson, L. D., Zavagno, A., Deharveng, L., et al. 2012, A\&A, 542, A10

Anderson, L. D., Deharveng, L., Zavagno, A., et al. 2015, ApJ, 800, 101

Araya, E., Hofner, P., Kurtz, S., Bronfman, L., \& DeDeo, S. 2005, ApJS, 157, 279

Beichman, C. A., Neugebauer, G., Habing, H. J., Clegg, P. E., \& Chester, T. J., eds. 1988, Infrared astronomical satellite (IRAS) catalogs and atlases, (Singapore: IRAS) 1

Benjamin, R. A., Churchwell, E., Babler, B. L., et al. 2003, PASP, 115, 953

Bloemen, J. B. G. M., Strong, A. W., Mayer-Hasselwander, H. A., et al. 1986, A\&A, 154, 25

Bock, D. C. J., Large, M. I., \& Sadler, E. M. 1999, AJ, 117, 1578

Borissova, J., Bonatto, C., Kurtev, R., et al. 2011, A\&A, 532, A131

Bronfman, L., Nyman, L. A., \& May, J. 1996, A\&AS, 115, 81

Cappa, C. E., Duronea, N., Firpo, V., et al. 2016, A\&A, 585, A30

Carpenter, J. M., Hillenbrand, L. A., \& Skrutskie, M. F. 2001, AJ, 121, 3160

Caswell, J. L. 1998, MNRAS, 297, 215

Caswell, J. L. 2009, PASA, 26, 454

Chaisson, E. J. 1976, in Frontiers of Astrophysics, ed. E. H. Avrett (Berlin: Springer), 259

Churchwell, E., Povich, M. S., Allen, D., et al. 2006, ApJ, 649, 759

Churchwell, E., Watson, D. F., Povich, M. S., et al. 2007, ApJ, 670, 428

Clemens, D. P. 1985, ApJ, 295, 422 
Cohen, R. S., Grabelsky, D. A., May, J., et al. 1985, ApJ, 290, L15 Comito, C., Schilke, P., Phillips, T. G., et al. 2005, ApJS, 156, 127 Cousins, A. W. J. 1978, The Observatory, 98, 54

Dedes, C., Leurini, S., Wyrowski, F., et al. 2011, A\&A, 526, A59

Deharveng, L., Lefloch, B., Kurtz, S., et al. 2008, A\&A, 482, 585

Deharveng, L., Zavagno, A., Schuller, F., et al. 2009, A\&A, 496, 177

Devine, K., Mori, J., Watson, C., Trujillo, L., \& Hicks, M. 2018, ApJ, 861, 117

Digel, S. W., Grenier, I. A., Heithausen, A., Hunter, S. D., \& Thaddeus, P. 1996, ApJ, 463, 609

Ducati, J. R., Bevilacqua, C. M., Rembold, S. r. B., \& Ribeiro, D. 2001, ApJ, 558,309

Dumke, M., \& Mac-Auliffe, F. 2010, SPIE Conf. Ser. 7737, 77371J

Duronea, N. U., Cappa, C. E., Bronfman, L., et al. 2017, A\&A, 606, A8

Duronea, N. U., Vasquez, J., Gómez, L., et al. 2015, A\&A, 582, A2

Egan, M. P., Price, S. D., \& Kraemer, K. E. 2003, BAAS, 35, 1301

Elmegreen, B. G., \& Lada, C. J. 1977, ApJ, 214, 725

Faúndez, S., Bronfman, L., Garay, G., et al. 2004, A\&A, 426, 97

Figueira, M., Zavagno, A., Deharveng, L., et al. 2017, A\&A, 600, A93

Foreman-Mackey, D., Hogg, D. W., Lang, D., \& Goodman, J. 2013, PASP, 125, 306

Frerking, M. A., Langer, W. D., \& Wilson, R. W. 1982, ApJ, 262, 590

Gaia Collaboration (Prusti, T., et al.) 2016, A\&A, 595, A1

Gaia Collaboration (Brown, A. G. A., et al.) 2018, A\&A, 616, A1

Güsten, R., Nyman, L. Å., Schilke, P., et al. 2006, A\&A, 454, L13

Gutermuth, R. A., Megeath, S. T., Myers, P. C., et al. 2009, ApJS, 184, 18

Henden, A. A., Terrell, D., Welch, D., \& Smith, T. C. 2010, BAAS, 42, 470.11

Henning, T., Michel, B., \& Stognienko, R. 1995, Planet. Space Sci., 43, 1333

Henning, T., Lapinov, A., Schreyer, K., Stecklum, B., \& Zinchenko, I. 2000, A\&A, 364, 613

Hildebrand, R. H. 1983, QJRAS, 24, 267

Hill, T., Burton, M. G., Minier, V., et al. 2005, MNRAS, 363, 405

Hunter, S. D., Bertsch, D. L., Catelli, J. R., et al. 1997, ApJ, 481, 205

Inoue, A. K. 2001, AJ, 122, 1788

Junkes, N., Fuerst, E., \& Reich, W. 1992, A\&A, 261, 289

Kendrew, S., Beuther, H., Simpson, R., et al. 2016, ApJ, 825, 142

Kharchenko, N. V., \& Roeser, S. 2009, VizieR Online Data Catalog: I/280B

Koenig, X. P., Leisawitz, D. T., Benford, D. J., et al. 2012, ApJ, 744, 130

König, C., Urquhart, J. S., Csengeri, T., et al. 2017, A\&A, 599, A139

Koornneef, J. 1983, A\&A, 128, 84

Kurtz, S., Cesaroni, R., Churchwell, E., Hofner, P., \& Walmsley, C. M. 2000 in Protostars and Planets IV, ed. V. Mannings, A. P. Boss, \& S. S. Russell (Tucson: University of Arizona Press), 299

Langer, W. D., \& Penzias, A. A. 1993, ApJ, 408, 539
Lefloch, B., \& Lazareff, B. 1994, A\&A, 289, 559

Liu, H.-L., Wu, Y., Li, J., et al. 2015, ApJ, 798, 30

Lumsden, S. L., Hoare, M. G., Oudmaijer, R. D., \& Richards, D. 2002, MNRAS, 336,621

MacLeod, G. C., Scalise, E. J., Saedt, S., Galt, J. A., \& Gaylard, M. J. 1998, AJ 116,1897

Martins, F., Schaerer, D., \& Hillier, D. J. 2005, A\&A, 436, 1049

McClure-Griffiths, N. M., Dickey, J. M., Gaensler, B. M., et al. 2005, ApJS, 158, 178

Miettinen, O., Harju, J., Haikala, L. K., \& Pomrén, C. 2006, A\&A, 460, 721

Molinari, S., Swinyard, B., Bally, J., et al. 2010, A\&A, 518, L100

Möller, T., Endres, C., \& Schilke, P. 2017, A\&A, 598, A7

Ossenkopf, V., \& Henning, T. 1994, A\&A, 291, 943

Osterloh, M., Henning, T., \& Launhardt, R. 1997, ApJS, 110, 71

Panagia, N., \& Walmsley, C. M. 1978, A\&A, 70, 411

Parker, Q. A., Phillipps, S., Pierce, M. J., et al. 2005, MNRAS, 362, 689

Povich, M. S., Stone, J. M., Churchwell, E., et al. 2007, ApJ, 660, 346

Preibisch, T., Roccatagliata, V., Gaczkowski, B., \& Ratzka, T. 2012, A\&A, 541, A132

Reid, M. J., Menten, K. M., Brunthaler, A., et al. 2014, ApJ, 783, 130

Reynoso, E. M., Cichowolski, S., \& Walsh, A. J. 2017, MNRAS, 464, 3029

Rieke, G. H., \& Lebofsky, M. J. 1985, ApJ, 288, 618

Rohlfs, K., \& Wilson, T. L. 2004, Tools of radio astronomy (Berlin: Springer)

Samal, M. R., Zavagno, A., Deharveng, L., et al. 2014, A\&A, 566, A122

Schmidt-Kaler. Th. 1982, in Landolt-Bornstein New Series, Group VI, eds. K. Schaifers, \& H. H. Voigt (Berlin: Springer-Verlag), 2b

Schuller, F., Menten, K. M., Contreras, Y., et al. 2009, A\&A, 504, 415

Skrutskie, M. F., Cutri, R. M., Stiening, R., et al. 2006, AJ, 131, 1163

Solomon, P. M., Rivolo, A. R., Barrett, J., \& Yahil, A. 1987, ApJ, 319, 730

Strong, A. W., \& Mattox, J. R. 1996, A\&A, 308, L21

Tenorio-Tagle, G. 1979, A\&A, 71, 59

Urquhart, J. S., Busfield, A. L., Hoare, M. G., et al. 2007, A\&A, 461, 11

Vassilev, V., Meledin, D., Lapkin, I., et al. 2008, A\&A, 490, 1157

Walch, S., Whitworth, A. P., Bisbas, T. G., Hubber, D. A., \& Wünsch, R. 2015, MNRAS, 452, 2794

Watson, C., Povich, M. S., Churchwell, E. B., et al. 2008, ApJ, 681, 1341

Wenger, T. V., Balser, D. S., Anderson, L. D., \& Bania, T. M. 2018, ApJ, 856, 52

Whitworth, A. P., Bhattal, A. S., Chapman, S. J., Disney, M. J., \& Turner, J. A. 1994, MNRAS, 268, 291

Wright, E. L., Eisenhardt, P. R. M., Mainzer, A. K., et al. 2010, AJ, 140, 1868

Yamaguchi, R., Akira, M., \& Yasuo, F. 1999, in Star Formation 1999, ed. T. Nakamoto (Cambridge: Cambridge University Press), 383

Zavagno, A., Deharveng, L., Comerón, F., et al. 2006, A\&A, 446, 171

Zinchenko, I., Henkel, C., \& Mao, R. Q. 2000, A\&A, 361, 1079 
Appendix A: Identified candidate YSOs

Table A.1. YSO candidates obtained from MSX, WISE, and Spitzer catalogs.

\begin{tabular}{|c|c|c|c|c|c|c|c|c|}
\hline \multicolumn{9}{|c|}{ MSX sources } \\
\hline$\#$ & Designation & $\begin{array}{c}\alpha \\
{[\mathrm{h} \mathrm{m} \mathrm{s}]}\end{array}$ & $\begin{array}{c}\delta \\
{[\circ, \prime \prime]}\end{array}$ & $\begin{array}{l}\mathrm{F}_{8} \\
{[\mathrm{Jy}]}\end{array}$ & $\begin{array}{l}\mathrm{F}_{12} \\
{[\mathrm{Jy}]}\end{array}$ & $\begin{array}{l}\mathrm{F}_{14} \\
{[\mathrm{Jy}]}\end{array}$ & $\begin{array}{l}\mathrm{F}_{21} \\
{[\mathrm{Jy}]}\end{array}$ & $\begin{array}{l}\text { Notes } \\
\text { (class, match with other YSO and } \\
\text { with }{ }^{13} \mathrm{CO} / \mathrm{CO} \text { or IR structure) } \\
\end{array}$ \\
\hline 1 & G301.1364-00.2249 & 123535.2 & -630230 & 1.41 & 5.30 & 17.92 & 120.23 & (MYSO) $\mathrm{MC}$ \\
\hline 2 & G301.130c & 353 & -630 & 2.216 & 2.702 & 1.477 & 8.930 & (CHII) MC3, WISE \\
\hline \multicolumn{9}{|c|}{ WISE sources } \\
\hline$\#$ & Designation & $\begin{array}{c}\alpha \\
{[\mathrm{h} \mathrm{m} \mathrm{s}]}\end{array}$ & $\begin{array}{c}\delta \\
{[0,1 /]}\end{array}$ & $\begin{array}{c}\text { W1 } \\
{[\mathrm{mag}]}\end{array}$ & $\begin{array}{c}\mathrm{W} 2 \\
{[\mathrm{mag}]}\end{array}$ & $\begin{array}{c}\text { W3 } \\
{[\mathrm{mag}]}\end{array}$ & $\begin{array}{c}\text { W4 } \\
{[\mathrm{mag}]}\end{array}$ & \\
\hline 3 & $\mathrm{~J} 123531$. & 123531.5 & -630306.4 & 8.759 & 7.615 & 3.207 & -0.535 & (Class I), MC3, \\
\hline 4 & J123529.88-630 417.1 & 123529.9 & -630417.1 & 11.847 & 10.812 & 5.958 & 2.858 & (Class I), MC2 \\
\hline 5 & $\mathrm{~J} 123553.78-630012.6$ & 123553.8 & -630012.6 & 9.87 & 8.769 & 5.207 & 1.367 & $($ Class I), $(+)$ \\
\hline 6 & $\mathrm{~J} 123535.13-630230.3$ & 123535.1 & -630230.3 & 9.49 & 6.629 & 1.011 & -3.604 & (Class I), MC3, MSX \\
\hline 7 & $\mathrm{~J} 123534.52-630147.8$ & 123534.5 & -630147.8 & 11.804 & 10.715 & 5.25 & 2.989 & (Class I), MC3, (*) \\
\hline 8 & J123529.71-630 425.9 & 123529.7 & -630425.9 & 10.829 & 9.987 & 6.386 & 4.376 & (Class II), $\mathrm{N}$ \\
\hline 9 & J123528.94-630 432.6 & 123528.9 & -630432.6 & 10.492 & 9.912 & 6.113 & 3.56 & (Class II), MC2 \\
\hline 10 & $\mathrm{~J} 123531.19-630328.3$ & 123531.2 & -630328.3 & 10.324 & 9.984 & 6.269 & 3.648 & (Class II), M \\
\hline 11 & $\mathrm{~J} 123538.44-630318.5$ & 123538.5 & -630318.5 & 13.115 & 12.557 & 6.902 & 3.138 & (Class II), $\mathrm{N}$ \\
\hline 12 & $\mathrm{~J} 123537.22-630300.1$ & 123537.2 & -630300.1 & 11.906 & 10.999 & 8.069 & 1.563 & (Class II), I \\
\hline 13 & $\mathrm{~J} 123534.55-630311.1$ & 123534.6 & -630311.1 & 11.405 & 10.717 & 5.906 & 3.999 & (Class II), \\
\hline 14 & $\mathrm{~J} 123628.80-630500.5$ & 23628.8 & -630500.5 & 10.379 & 9.698 & 6.464 & 4.797 & (Class II), Feature 2, \\
\hline 15 & $\mathrm{~J} 123606.78-630458.5$ & 23606.8 & -630458.5 & 10.812 & 10.396 & 9.114 & 6.107 & (Class II) \\
\hline 16 & 5717.3 & 2355 & -625717.3 & 12.834 & 12.427 & 5.898 & 3.722 & \\
\hline 17 & $\mathrm{~J} 123556.7$ & 123556.7 & -625804.3 & 13.196 & 12.637 & 6.007 & 4.376 & $(\mathrm{Cl}$ \\
\hline 18 & $\mathrm{~J} 123556.58-630058.1$ & 23556.6 & -630058.1 & 12.681 & 12.309 & 6.877 & 5.598 & (Class II), MC5, $(*)$ \\
\hline 19 & $\mathrm{~J} 123559.25-630043.5$ & 3559.3 & -630043.5 & 12.784 & 12.065 & 6.416 & 4.826 & (Class II), MC5, $(*)$ \\
\hline 20 & $\mathrm{~J} 123605.26-630000.2$ & 3605.3 & -630000.2 & 12.44 & 11.992 & 7.689 & 5.894 & (Class II), MC5, $(*)$ \\
\hline 21 & & & & 13.621 & & & & \\
\hline 22 & J123544.39-630 151.3 & 123544.4 & -630151.3 & 12.362 & 11.598 & 7.181 & 4.462 & (Class II), MC3, $(*)$ \\
\hline 23 & J123535.61-630 129.4 & 123535.6 & -630129.4 & 11.64 & 11.000 & 5.101 & 3.019 & (Class II), MC3, (+) \\
\hline 24 & $\mathrm{~J} 123533.44-630130.5$ & 123533.4 & -630130.5 & 11.504 & 10.981 & 5.308 & 2.927 & (Class II), MC3, (+) \\
\hline 25 & & & & & & & & \\
\hline 26 & $\mathrm{~J} 123542.44-630148.3$ & 123542.3 & -630148.3 & 11.042 & 10.237 & 6.363 & 2.654 & (Class II), MC3, (*), Spitzer \#67 \\
\hline 27 & J123538.39-630 139.4 & 123538.4 & -630139.4 & 10.882 & 10.354 & 5.752 & 2.963 & (Class II), MC3, (+) \\
\hline 28 & $\mathrm{~J} 123525.27-630114.3$ & 123525.3 & -630114.3 & 9.593 & 8.952 & 4.275 & 2.262 & (Class II), MC3, (+) \\
\hline 29 & $\mathrm{~J} 123528.77-630113.8$ & 123528.8 & -630113.8 & 10.472 & 9.535 & 4.4 & 2.781 & (Class II), MC3, (+) \\
\hline 30 & $\mathrm{~J} 123522.97-630048.0$ & 123523.0 & -630048.0 & 11.545 & 10.94 & 6.364 & 1.844 & (Class II), \\
\hline 31 & J123526.73-630 156.8 & 123526.7 & -630156.8 & 11.679 & 10.821 & 5.627 & 2.865 & (Class II), MC3 \\
\hline 32 & $\mathrm{~J} 123521.24-630158.0$ & 123521.2 & -630158.0 & 11.983 & 11.316 & 5.168 & 2.302 & (Class II), MC3, \\
\hline 33 & $\mathrm{~J} 123525.41-630204.4$ & 123525.4 & -630204.4 & 11.457 & 10.729 & 5.582 & & (Class II), MC3 \\
\hline 34 & $\mathrm{~J} 123524.65-630218.0$ & 123524.7 & -630218.0 & 11.548 & 10.785 & 5.839 & 3.296 & (Class II), \\
\hline 35 & $\mathrm{~J} 123525.28-630126.8$ & 3525.3 & -630126.8 & 10.764 & 10.072 & 5.26 & 4.748 & (Class II), MC3, (+) \\
\hline 36 & J123552.92-625938.6 & 123552.9 & -625938.6 & 13.013 & 12.391 & 6.458 & 4.429 & (Class II), (+) \\
\hline 37 & $\mathrm{~J} 123546.50-630010.4$ & 123546.5 & -630010.4 & 13.483 & 12.56 & 6.118 & 4.25 & (Class II), (+) \\
\hline 38 & $\mathrm{~J} 123551.36-630013.5$ & 123551.4 & -630013.5 & 12.246 & 11.293 & 5.803 & 4.145 & (Class II), \\
\hline 39 & $\mathrm{~J} 123550$ & & 01.0 & & & & & \\
\hline 40 & J123541.99-630 100.8 & 123542.0 & -630100.8 & 11.681 & 10.913 & 4.941 & 3.329 & (Class II), (+) \\
\hline 41 & $\mathrm{~J} 123543.50-630002.3$ & 123543.5 & -630002.3 & 13.039 & 12.656 & 7.897 & 4.866 & (Class II), (+) \\
\hline 42 & $\mathrm{~J} 123543.44-630013.6$ & 123543.4 & -630013.6 & 12.344 & 12.003 & 6.657 & 4.019 & (Class II), (+) \\
\hline 43 & $\mathrm{~J} 123542.11-630037.9$ & 123542.1 & -630037.9 & 12.257 & 11.573 & 5.277 & 3.368 & (Class II), (+) \\
\hline 44 & $\mathrm{~J} 123543.69-630050.6$ & 123543.7 & -630050.6 & 11.771 & 11.174 & 5.177 & 3.43 & (Class II), (+) \\
\hline 45 & $\mathrm{~J} 123545.43-630023.2$ & 123545.4 & -630023.2 & 11.848 & 11.265 & 5.344 & 3.169 & (Class II), (+) \\
\hline 46 & $\mathrm{~J} 123544.72-630038.4$ & 123544.7 & -630038.4 & 12.206 & 11.608 & 5.446 & 4.213 & (Class II), (+) \\
\hline 47 & $\mathrm{~J} 123543.60-630027.6$ & 123543.6 & -630027.6 & 12.647 & 12.25 & 6.496 & 5.909 & (Class II), (+) \\
\hline 48 & $\mathrm{~J} 123544.53-625720.1$ & 123544.5 & -625720.1 & 7.801 & 6.65 & 5.019 & 4.336 & (Class II), Spitzer \#52 \\
\hline 49 & $\mathrm{~J} 123454.60-630009.5$ & 123454.6 & -630009.5 & 11.662 & 11.15 & 8.66 & 6.146 & (Class II), Feature 3, Spitzer $\# 72$ \\
\hline 50 & J123558.47-625 603.9 & 123558.5 & -625603.9 & 10.983 & 10.688 & 7.725 & 5.376 & (Class II) \\
\hline
\end{tabular}


Table A.1. continued.

\begin{tabular}{|c|c|c|c|c|c|c|c|c|}
\hline \multicolumn{9}{|c|}{ Spitzer sources } \\
\hline$\overline{\#}$ & Designation & $\begin{array}{c}\alpha \\
{[\mathrm{h} \mathrm{m} \mathrm{s}]}\end{array}$ & $\begin{array}{c}\delta \\
{[0 / \prime \prime]}\end{array}$ & $\begin{array}{c}3.6 \mu \mathrm{m} \\
{[\mathrm{mag}]}\end{array}$ & $\begin{array}{c}4.5 \mu \mathrm{m} \\
{[\mathrm{mag}]}\end{array}$ & $\begin{array}{c}5.8 \mu \mathrm{m} \\
{[\mathrm{mag}]}\end{array}$ & $\begin{array}{c}8.0 \mu \mathrm{m} \\
{[\mathrm{mag}]}\end{array}$ & \\
\hline 51 & G301.1340-00.0894 & 123538.3 & -625421.5 & 12.44 & 11.532 & 10.577 & 9.693 & (Class I) \\
\hline 52 & G301.1489-00.1383 & 123544.5 & -625720.5 & 8.62 & 7.55 & 6.595 & 5.873 & (Class I), WISE \#48 \\
\hline 53 & G301.1866-00.1701 & 123603.4 & -625923.0 & 12.424 & 11.565 & 10.773 & 9.521 & (Class I) \\
\hline 54 & G301.1955-00.2012 & 123607.1 & -630116.6 & 11.595 & 10.529 & 9.709 & 8.791 & (Class I), MC5, $(*)$ \\
\hline 55 & G301.1766-00.2110 & 123556.8 & -630147.8 & 14.545 & 12.909 & 11.945 & 12.002 & (Class I), MC4 \\
\hline 56 & G301.2000-00.1534 & 123611.0 & -625825.8 & 13.885 & 12.788 & 11.921 & 10.916 & (Class I) \\
\hline 57 & G301.1338-00.2711 & 123532.2 & -630514.4 & 12.721 & 11.54 & 10.612 & 9.959 & (Class I), MC1 \\
\hline 58 & G301.1402-00.2298 & 123537.0 & -630247.4 & 12.521 & 11.843 & 11.118 & 10.65 & (Class II), MC3 \\
\hline 59 & G301.1395-00.2303 & 123536.6 & -630249.0 & 11.273 & 10.604 & 10.012 & 9.482 & (Class II), MC3 \\
\hline 60 & G301.1523-00.2264 & 123543.5 & -630237.8 & 12.498 & 12.146 & 11.893 & 10.887 & (Class II), MC3 \\
\hline 61 & G301.2400-00.2609 & 123628.8 & -630500.6 & 10.204 & 9.729 & 9.231 & 7.869 & (Class II), Feature 2, WISE \#14 \\
\hline 62 & G301.1973-00.2637 & 123606.1 & -630501.6 & 11.215 & 10.808 & 10.571 & 10.131 & (Class II) \\
\hline 63 & G301.2169-00.2211 & 123617.8 & -630232.7 & 13.615 & 12.915 & 12.217 & 11.599 & (Class II), MC6 \\
\hline 64 & G301.1745-00.2102 & 123555.7 & -630144.5 & 13.464 & 12.670 & 12.148 & 10.998 & (Class II) \\
\hline 65 & G301.2153-00.1838 & 123618.1 & -630018.3 & 7.683 & 7.482 & 7.007 & 6.772 & (Class II), MC5, $(*)$ \\
\hline 66 & G301.1311-00.2271 & 123532.3 & -630235.7 & 12.992 & 11.898 & 11.354 & 10.504 & (Class II), MC3 \\
\hline 67 & G301.1500-00.2124 & 123542.7 & -630147.0 & 11.332 & 10.95 & 10.353 & 9.342 & (Class II), MC3, (*), WISE \#26 \\
\hline 68 & G301.1402-00.2155 & 123537.5 & -630156.0 & 11.273 & 10.637 & 10.065 & 9.522 & (Class II), MC3, $(*)$ \\
\hline 69 & G301.1432-00.2154 & 123539.9 & -630156.3 & 12.104 & 11.591 & 11.068 & 10.594 & (Class II), MC3, (*) \\
\hline 70 & G301.1090-00.1906 & 123521.8 & -630019.6 & 13.625 & 12.923 & 12.308 & 11.642 & (Class II) \\
\hline 71 & G301.1250-00.2201 & 123529.3 & -630209.2 & 10.166 & 9.538 & 8.947 & 8.425 & (Class II), MC3 \\
\hline 72 & G301.0569-00.1911 & 123454.3 & -630009.5 & 12.334 & 11.901 & 11.645 & 10.713 & (Class II), Feature 3 \\
\hline 73 & G301.0578-00.1910 & 123454.8 & -630009.4 & 12.095 & 11.671 & 11.336 & 10.556 & (Class II), Feature 3, WISE \#49 \\
\hline 74 & G301.1525-00.0712 & 123548.6 & -625320.2 & 12.940 & 12.224 & 11.714 & 11.15 & (Class II) \\
\hline
\end{tabular}

